\title{
DECORRELATION ESTIMATES FOR A 1D TIGHT BINDING MODEL IN THE LOCALIZED REGIME
}

\author{
TRINH TUAN PHONG
}

\begin{abstract}
In this article, we prove decorrelation estimates for the eigenvalues of a $1 \mathrm{D}$ discrete tight binding model near two distinct energies in the localized regime. Consequently, for any integer $n \geq 2$, the asymptotic independence for local level statistics near $n$ distinct energies is obtained.
\end{abstract}

\section{INTRODUCTION}

The present paper deals with the following lattice Hamiltonian with offdiagonal disorder in dimension 1: for $u=\{u(n)\}_{n \in \mathbb{Z}} \in l^{2}(\mathbb{Z})$, set

$$
\left(H_{\omega} u\right)(n)=\omega_{n}(u(n)-u(n+1))-\omega_{n-1}(u(n-1)-u(n)) .
$$

The model (1.1) appears in the description of waves (light, acoustic waves, etc) which propagate through a disordered, discrete medium (c.f. [1]). We can see $\left\{\omega_{n}\right\}_{n \in \mathbb{Z}}$ in this model as weights of bonds of the lattice $\mathbb{Z}$.

Throughout this article, we assume that $\omega:=\left\{\omega_{n}\right\}_{n \in \mathbb{Z}}$ are non-negative i.i.d. random variables (r.v.'s for short) with a bounded, compactly supported density $\rho$.

In addition, from Section 1 to Section 3 , we assume more that $\omega_{n} \in\left[\alpha_{0}, \beta_{0}\right]$ for all $n \in \mathbb{Z}$ where $\beta_{0}>\alpha_{0}>0$. In Section 4 , we will comment on relaxing the hypothesis of the lower bound of r.v.'s $\omega$.

It is known that (see [5]):

- the operator $H_{\omega}$ admits an almost sure spectrum $\Sigma:=\left[0,4 \beta_{0}\right]$.

- $H_{\omega}$ has an integrated density of states defined as follows: $\omega$-a.s., the following limit exists and is $\omega$ independent:

$$
N(E):=\lim _{|\Lambda| \rightarrow+\infty} \frac{\#\left\{\text { e.v. of } H_{\omega} \text { less than } \mathrm{E}\right\}}{|\Lambda|} \text { for a.e. } E .
$$

As a direct consequence of the Wegner estimate (see Theorem 2.1 in Section 2), $N(E)$ is defined everywhere in $\mathbb{R}$ and absolutely continuous w.r.t. Lebesgue measure with a bounded derivative $\nu(E)$ called the density of states of $H_{\omega}$.

In the present paper, we follow a usual way to study various statistics related to random operators. We restrict the operator $H_{\omega}$ on some interval $\Lambda \subset \mathbb{Z}$ of finite length with some boundary condition and obtain a finite-volume

Date: April 30, 2018. 
operator which is denoted by $H_{\omega}(\Lambda)$. Then, we study diverse statistics for this operator in the limit when $|\Lambda|$ goes to infinity.

Throughout this paper, the boundary condition to define $H_{\omega}(\Lambda)$ is always the periodic boundary condition. For example, if $\Lambda=[1, N]$, the operator $H_{\omega}(\Lambda)$ is a symmetric $N \times N$ matrix of the following form:

$$
\left(\begin{array}{cccccc}
\omega_{N}+\omega_{1} & -\omega_{1} & 0 & \ldots & 0 & -\omega_{N} \\
-\omega_{1} & \omega_{1}+\omega_{2} & -\omega_{2} & \ldots & 0 & 0 \\
\vdots & \vdots & \vdots & \ldots & \vdots & \vdots \\
0 & 0 & 0 & \ldots & \omega_{N-2}+\omega_{N-1} & -\omega_{N-1} \\
-\omega_{N} & 0 & 0 & \ldots & -\omega_{N-1} & \omega_{N-1}+\omega_{N}
\end{array}\right)
$$

For $L \in \mathbb{N}$, let $\Lambda=\Lambda_{L}:=[-L, L]$ be a large interval in $\mathbb{Z}$ and $|\Lambda|:=(2 L+1)$ be its cardinality.

We will denote the eigenvalues of $H_{\omega}(\Lambda)$ ordered increasingly and repeated according to multiplicity by $E_{1}(\omega, \Lambda) \leqslant E_{2}(\omega, \Lambda) \leqslant \cdots \leqslant E_{|\Lambda|}(\omega, \Lambda)$.

Let $I$ be the localized regime (the region of localization) in $\Sigma$ where the finite-volume fractional-moment criteria for localization are satisfied for the finite-volume operators $H_{\omega}(\Lambda)$ when $|\Lambda|$ is large enough (see Section 2 and [2] for more details). In this region, the spectrum of $H_{\omega}$ is pure point and the corresponding eigenfunctions decay exponentially at infinity.

Pick $E$ a positive energy in $I$ with $\nu(E)>0$, and define the local level statistics near $E$ as follows

$$
\Xi(\xi, E, \omega, \Lambda)=\sum_{n=1}^{|\Lambda|} \delta_{\xi_{n}}(E, \omega, \Lambda)(\xi)
$$

where

$$
\xi_{n}(E, \omega, \Lambda)=|\Lambda| \nu(E)\left(E_{n}(\omega, \Lambda)-E\right) .
$$

For the model (1.1), it is known that the weak limit of the above point process is a Poisson point process:

Theorem 1.1. [5]] Assume that $E$ is a positive energy in $I$ with $\nu(E)>0$. Then, when $|\Lambda| \rightarrow+\infty$, the point process $\Xi(\xi, E, \omega, \Lambda)$ converges weakly to a Poisson point process with the intensity 1 i.e., for $\left(U_{j}\right)_{1 \leqslant j \leqslant J}, U_{j} \subset \mathbb{R}$ bounded measurable and $U_{j^{\prime}} \cap U_{j}=\emptyset$ if $j \neq j^{\prime}$ and $\left(k_{j}\right)_{1 \leqslant j \leqslant J} \in \mathbb{N}^{J}$, we have

$$
\lim _{|\Lambda| \rightarrow+\infty}\left|\mathbb{P}\left(\left\{\begin{array}{cc}
\#\left\{j ; \xi_{j}(E, \omega, \Lambda) \in U_{1}\right\} & =k_{1} \\
\vdots & \vdots \\
\#\left\{j ; \xi_{j}(E, \omega, \Lambda) \in U_{j}\right\} & =k_{J}
\end{array}\right\}\right)-\prod_{j=1}^{J} \frac{\left|U_{j}\right|^{k_{j}}}{k_{j} !} e^{-\left|U_{j}\right|}\right|=0 .
$$

Recently, for the 1D discrete Anderson model, Klopp [8] showed more that if we pick two fixed, distinct energies $E$ and $E^{\prime}$ in the localized regime, their two corresponding point processes $\Xi(\xi, E, \omega, \Lambda)$ and $\Xi\left(\xi, E^{\prime}, \omega, \Lambda\right)$ converge weakly, respectively to two independent Poisson point processes. In other words, the limits of $\Xi(\xi, E, \omega, \Lambda)$ and $\Xi\left(\xi, E^{\prime}, \omega, \Lambda\right)$ are stochastically independent. 
It is known that the above statement holds true if one can prove a so-called decorrelation estimate.

That is exactly what we want to carry out here for the $1 \mathrm{D}$ discrete lattice Hamiltonian with off-diagonal disorder (1.1). Our decorrelation estimate is the following:

Theorem 1.2. Let $E, E^{\prime}$ be two positive, distinct energies in the localized regime. Pick $\beta \in(1 / 2,1)$ and $\alpha \in(0,1)$. Then, for any $c>0$, there exists $C>0$ such that, for $L$ large enough and $c L^{\alpha} \leqslant l \leqslant L^{\alpha} / c$, one has

$$
\mathbb{P}\left(\left\{\begin{array}{c}
\sigma\left(H_{\omega}\left(\Lambda_{l}\right)\right) \cap\left(E+L^{-1}(-1,1)\right) \neq \emptyset \\
\sigma\left(H_{\omega}\left(\Lambda_{l}\right)\right) \cap\left(E^{\prime}+L^{-1}(-1,1)\right) \neq \emptyset
\end{array}\right\}\right) \leqslant C(l / L)^{2} e^{(\log L)^{\beta}} .
$$

This decorrelation estimate means that, up to a sub-polynomial error, the probability of obtaining simultaneously two eigenvalues near two distinct energies is bounded by the product of the estimates given by Wegner estimate for each of these two energies. Roughly speaking, two eigenvalues of our model near two distinct energies behave like two independent random variables.

Thanks to Theorem 1.2, we can proceed as in Section 3 of [8] to obtain the asymptotic independence of the weak limits of $\Xi(\xi, E, \omega, \Lambda)$ and $\Xi\left(\xi, E^{\prime}, \omega, \Lambda\right)$ with $E, E^{\prime}>0$ for the model (1.1):

Theorem 1.3. Pick two positive, distinct energies $E$ and $E^{\prime}$ in the localized regime such that $\nu(E)>0$ and $\nu\left(E^{\prime}\right)>0$.

When $|\Lambda| \rightarrow+\infty$, the point processes $\Xi(\xi, E, \omega, \Lambda)$, and $\Xi\left(\xi, E^{\prime}, \omega, \Lambda\right)$ converge weakly respectively to two independent Poisson processes on $\mathbb{R}$ with intensity the Lebesgue measure.

That is, for $\left(U_{j}\right)_{1 \leqslant j \leqslant J}, U_{j} \subset \mathbb{R}$ bounded measurable and $U_{j^{\prime}} \cap U_{j}=\emptyset$ if $j \neq j^{\prime}$ and $\left(k_{j}\right)_{1 \leqslant j \leqslant J} \in \mathbb{N}^{J}$ and $\left(U_{j}^{\prime}\right)_{1 \leqslant j \leqslant J^{\prime}}, U_{j}^{\prime} \subset \mathbb{R}$ bounded measurable and $U_{j^{\prime}}^{\prime} \cap U_{j}^{\prime}=\emptyset$ if $j \neq j^{\prime}$ and $\left(k_{j}^{\prime}\right)_{1 \leqslant j \leqslant J^{\prime}} \in \mathbb{N}^{J^{\prime}}$, we have

$$
\mathbb{P}\left(\begin{array}{cc}
\#\left\{j ; \xi_{j}(E, \omega, \Lambda) \in U_{1}\right\} & =k_{1} \\
\vdots & \vdots \\
\#\left\{j ; \xi_{j}(E, \omega, \Lambda) \in U_{j}\right\} & =k_{J} \\
\#\left\{j ; \xi_{j}\left(E^{\prime}, \omega, \Lambda\right) \in U_{1}^{\prime}\right\} & =k_{1}^{\prime} \\
\vdots & \vdots \\
\#\left\{j ; \xi_{j}\left(E^{\prime}, \omega, \Lambda\right) \in U_{J}^{\prime}\right\} & =k_{J}^{\prime}
\end{array}\right\} \rightarrow \prod_{j=1}^{J} \frac{\left|U_{j}\right|^{k_{j}}}{k_{j} !} e^{-\left|U_{j}\right|} \prod_{i=1}^{J^{\prime}} \frac{\left|U_{i}^{\prime}\right|_{i}^{\prime}}{k_{i}^{\prime} !} e^{-\left|U_{i}^{\prime}\right|}
$$

as $|\Lambda| \rightarrow+\infty$.

Moreover, in Section 5, we will generalize Theorem 1.3 by considering not only two but any fixed number of distinct energies.

To prove Theorem 1.2 for the model (1.1), we follow the strategy introduced in [8]. The key point of the proof of decorrelation estimates for the 1D discrete Anderson model in [8] is to derive that the gradient with respect to $\omega$ of two eigenvalues $E(\omega)$ and $E^{\prime}(\omega)$ near two distinct energies $E$ and $E^{\prime}$ 
are not co-linear with a good probability. In the discrete Anderson case, this statement will hold true if the gradients of $E(\omega)$ and $E^{\prime}(\omega)$ are distinct. In deed, the gradients of $E(\omega)$ and $E^{\prime}(\omega)$ have non-negative components and their $l^{1}$-norm are always equal to 1 . So, if they are co-linear, they should be the same.

Unfortunately, the $l^{1}$-norm of the gradient of an eigenvalue of our finitevolume operator $H_{\omega}(\Lambda)$ is not a constant w.r.t. $\omega$ anymore (it is even not bounded from below by a positive constant uniformly w.r.t. $\omega$ ). Moreover, to prove the above key point for the discrete Anderson model, [8] exploits the diagonal structure of the potential which can not be used for the present case. So, a different approach in the proof is needed to carry out Theorem 1.2. This approach is contained in Lemma 3.3.

In addition, Theorem 1.12 in [7] implies directly the following result for the model (1.1):

Theorem 1.4. [Theorem 1.12, [7]] Pick $0<E_{0} \in I$ such that the density of states $\nu$ is continuous and positive at $E_{0}$.

Consider two sequences of positive energies, say $\left(E_{\Lambda}\right)_{\Lambda},\left(E_{\Lambda}^{\prime}\right)_{\Lambda}$ such that

(1) $E_{\Lambda} \underset{\Lambda \rightarrow \mathbb{Z}^{d}}{\longrightarrow} E_{0}$ and $E_{\Lambda}^{\prime} \underset{\Lambda \rightarrow \mathbb{Z}^{d}}{\longrightarrow} E_{0}$,

(2) $\left|\Lambda \| N\left(E_{\Lambda}\right)-N\left(E_{\Lambda}^{\prime}\right)\right| \underset{\Lambda \rightarrow \mathbb{Z}^{d}}{\longrightarrow}+\infty$.

Then, the point processes $\Xi\left(\xi, E_{\Lambda}, \omega, \Lambda\right)$ and $\Xi\left(\xi, E_{\Lambda}^{\prime}, \omega, \Lambda\right)$ converges weakly respectively to two independent Poisson point processes in $\mathbb{R}$ with intensity the Lebesgue measure.

In Theorem 1.4, instead of fixing two distinct energies $E$ and $E^{\prime}$, one considers two sequences of positive energies $\left\{E_{\Lambda}\right\},\left\{E_{\Lambda}^{\prime}\right\}$ which tend to each other as $|\Lambda| \rightarrow \infty$. In addition, one assumes that, roughly speaking, there are sufficiently many eigenvalues of $H_{\omega}(\Lambda)$ between $E_{\Lambda}$ and $E_{\Lambda}^{\prime}$ as $\Lambda$ large. Then, the asymptotic independence of two point processes associated to $E_{\Lambda}$ and $E_{\Lambda}^{\prime}$ is obtained. (See Section 2 for the reason we only consider positive energies in the present paper).

Besides, it is known that the existence of an integrated density of states defined as in (1.2) implies that the average distance (mean spacing) between eigenlevels is of order $|\Lambda|^{-1}$.

Thus, according to Theorem 1.4, in the localized regime, eigenvalues separated by a distance that is asymptotically infinite with respect to the mean spacing between eigenlevels behave like independent random variables. In other words, there are no interactions between distinct eigenvalues, except at a very short distance.

Notation: In the present paper, we use Dirac's notations: If $\varphi$ is a vector in a Hilbert space $\mathcal{H}$, we denote by $|\varphi\rangle\langle\varphi|=\langle\varphi, \cdot\rangle_{\mathcal{H}} \varphi$ the projection operator on $\varphi$. Besides, throughout the present paper, the symbol $\|\cdot\|$ stands for the $l^{2}$-norm $\|\cdot\|_{2}$ in some finite dimensional Hilbert space. 


\section{Preliminaries}

We recall here a Wegner-type estimate and a Minami-type estimate for the model (1.1) which are essentially important for the proofs of Poisson statistics as well as those of decorrelation estimates.

Theorem 2.1. [Wegner estimate, Theorem 2.1, [5]]

$$
\mathbb{P}\left(\operatorname{dist}\left(E, \sigma\left(H_{\omega}(\Lambda)\right)\right) \leqslant \epsilon\right) \leq \frac{2 d\|s \rho(s)\|_{\infty}}{E-\epsilon} \epsilon|\Lambda|
$$

for all intervals $\Lambda \subset \mathbb{Z}$ and $0<\epsilon<E$.

Roughly speaking, the above Wegner estimate means that the probability that we can find an eigenvalue of the finite-volume operator $H_{\omega}(\Lambda)$ in a interval (suppose that the length of this interval is small) is bounded by the length of this interval times $|\Lambda|$.

Following is the Minami estimate:

Theorem 2.2. [Minami estimate, Theorem 3.1, [5]] There exists $C>0$ such that, for all intervals $J=[a, b] \subset(0,+\infty)$, and $\Lambda \subset \mathbb{Z}$, we have

$$
\mathbb{P}\left(\#\left\{\sigma\left(H_{\omega}(\Lambda)\right) \cap J\right\} \geqslant 2\right) \leqslant \beta_{0}\|\rho\|_{\infty}\|s \rho(s)\|_{\infty}(|J||\Lambda|)^{2} / 2 a^{2} .
$$

We should remark that although the almost sure spectrum $\Sigma$ of the operator (1.1) contains the point 0 (the bottom of $\Sigma$ ), the above Wegner and Minami estimates do not work at 0 . That is why, in the present paper, we will only consider energies who are strictly positive in $\Sigma$. Besides, in Theorem 2.2, the right hand side of the Minami estimate depends on the infimum of the interval $J$ which is different from the Minami estimate stated for the discrete Anderson model. However, this difference complicates nothing when we only consider energies close to a fixed, positive energy in the present paper.

Finally, we would like to remind readers of the precise definition of the localized regime associated to finite-volume operators.

Proposition 2.3. (c.f. [2], [8]) Let I be the region of $\Sigma$ where the finite volume fractional moment criteria of [2] for $H_{\omega}(\Lambda)$ are verified for $\Lambda$ sufficiently large.

Then, there exists $\nu>0$ such that, for any $p>0$, there exists $q>0$ and $L_{0}>0$ such that, for $L \geqslant L_{0}$, with probability larger than $1-L^{-p}$, if

(1) $\varphi_{n, \omega}$ is a normalized eigenvector of $H_{\omega}\left(\Lambda_{L}\right)$ associated to an energy $E_{n, \omega} \in I$,

(2) $x_{n, \omega} \in \Lambda_{L}$ is a maximum of $x \mapsto\left|\varphi_{n, \omega}(x)\right|$ in $\Lambda_{L}$,

then, for $x \in \Lambda_{L}$, one has

$$
\left|\varphi_{n, \omega}(x)\right| \leqslant L^{q} e^{-\nu\left|x-x_{n, \omega}\right|} .
$$

The point $x_{n, \omega}$ is called a localization center for $\varphi_{n, \omega}$ or $E_{n, \omega}$.

Remark that an eigenvalue of $H_{\omega}(\Lambda)$ can have more than one localization center. Nevertheless, it is not hard to check that, under assumptions of 
Proposition 2.3, all localization centers associated to one eigenvalue are contained in a disk of radius $C \log L$ with some constant $C$.

Hence, to define the localization center uniquely for an eigenvalue, we can order these centers lexicographically and choose the localization center associated to an eigenvalue to be the largest one.

\section{Proof of TheOREM 1.2}

Pick two distinct, positive energies $E, E^{\prime}$ in $I$ (the localized regime). Let $J_{L}=E+L^{-1}[-1,1]$ and $J_{L}^{\prime}=E^{\prime}+L^{-1}[-1,1]$ with $L$ large. We would like to begin this section by proving some elementary properties of eigenvalues of $H_{\omega}(\Lambda)$ with an arbitrary interval $\Lambda \in \mathbb{Z}$.

Lemma 3.1. Suppose that $\omega \mapsto E(\omega)$ is the only eigenvalue of $H_{\omega}(\Lambda)$ in $J_{L}$. Then

(1) $E(\omega)$ is simple and $\omega \mapsto E(\omega)$ is real analytic. Moreover, let $\omega \mapsto$ $\varphi(\omega)$ denote the real-valued, normalized eigenvector associated to $E(\omega)$, it is also real analytic in $\omega$.

(2) $\left\|\nabla_{\omega} E(\omega)\right\|_{1}=2 \sum_{\gamma \in \Lambda}\left\|\Pi_{\gamma} \varphi\right\|^{2}$ where $\Pi_{\gamma}=\frac{1}{2}\left|\delta_{\gamma}-\delta_{\gamma+1}\right\rangle\left\langle\delta_{\gamma}-\delta_{\gamma+1}\right|$ is a projection in $l^{2}(\Lambda)$. Besides, we have $E(\omega) \in\left[0,4 \beta_{0}\right]$.

(3) $\operatorname{Hess}_{\omega} E(\omega)=\left(h_{\gamma, \beta}\right)_{\gamma, \beta}$ where

- $h_{\gamma, \beta}:=-4\left\langle\left(H_{\omega}(\Lambda)-E(\omega)\right)^{-1} \psi_{\gamma}, \psi_{\beta}\right\rangle$.

- $\psi_{\gamma}:=\left\langle\Pi_{\gamma} \varphi, \varphi\right\rangle \varphi-\Pi_{\gamma} \varphi=-\Pi_{\langle\varphi\rangle^{\perp}}\left(\Pi_{\gamma} \varphi\right)$ where $\Pi_{\langle\varphi\rangle^{\perp}}$ is the orthogonal projection on $\langle\varphi\rangle^{\perp}$.

Proof of Lemma 3.1. (1) is true from the standard perturbation theory (c.f. [9]).

Now we will prove (2). Starting from the eigenequation

$$
H_{\omega}(\Lambda) \varphi=E(\omega) \varphi
$$

we have, for all $\gamma \in \Lambda$,

$$
\begin{aligned}
\partial_{\omega_{\gamma}} E(\omega) & =\left\langle\partial_{\omega_{\gamma}}\left(H_{\omega}(\Lambda) \varphi\right), \varphi\right\rangle+\left\langle H_{\omega}(\Lambda) \varphi, \partial_{\omega_{\gamma}} \varphi\right\rangle \\
& =\left\langle\partial_{\omega_{\gamma}}\left(H_{\omega}(\Lambda)\right) \varphi, \varphi\right\rangle+\left\langle H_{\omega}(\Lambda) \partial_{\omega_{\gamma}} \varphi, \varphi\right\rangle+\left\langle H_{\omega}(\Lambda) \varphi, \partial_{\omega_{\gamma}} \varphi\right\rangle \\
& =\left\langle\partial_{\omega_{\gamma}}\left(H_{\omega}(\Lambda)\right) \varphi, \varphi\right\rangle+\left\langle\partial_{\omega_{\gamma}} \varphi, H_{\omega}(\Lambda) \varphi\right\rangle+\left\langle H_{\omega}(\Lambda) \varphi, \partial_{\omega_{\gamma}} \varphi\right\rangle \\
& =\left\langle\partial_{\omega_{\gamma}}\left(H_{\omega}(\Lambda)\right) \varphi, \varphi\right\rangle+E(\omega)\left(\left\langle\partial_{\omega_{\gamma}} \varphi, \varphi\right\rangle+\left\langle\varphi, \partial_{\omega_{\gamma}} \varphi\right\rangle\right)
\end{aligned}
$$

where the last two equalities come from the symmetry of $H_{\omega}(\Lambda)$ and (3.1). Noting that

$$
\left\langle\partial_{\omega_{\gamma}} \varphi, \varphi\right\rangle+\left\langle\varphi, \partial_{\omega_{\gamma}} \varphi\right\rangle=2 \partial_{\omega_{\gamma}}\|\varphi\|^{2}=0
$$

Hence,

$$
\partial_{\omega_{\gamma}} E(\omega)=\left\langle\partial_{\omega_{\gamma}}\left(H_{\omega}(\Lambda)\right) \varphi, \varphi\right\rangle=2\left\langle\Pi_{\gamma} \varphi, \varphi\right\rangle .
$$

On the other hand, it is easy to check that $\Pi_{\gamma}=\Pi_{\gamma}^{*}=\Pi_{\gamma}^{2}$. Hence, $\Pi_{\gamma}$ is an orthogonal projection and $\partial_{\omega_{\gamma}} E(\omega)=2\left\|\Pi_{\gamma} \varphi\right\|^{2}$. 
Thanks to (3.1) and (3.2), we have the following important equality:

$$
\sum_{\gamma \in \Lambda} \omega_{\gamma} \partial_{\omega_{\gamma}} E(\omega)=2 \sum_{\gamma \in \Lambda} \omega_{\gamma}\left\langle\Pi_{\gamma} \varphi, \varphi\right\rangle=E(\omega)
$$

which characterize the form of our operator.

From (3.3) and $\|\varphi\|=1$, we infer that

$$
0 \leq E(\omega)=\sum_{\gamma \in \Lambda} \omega_{\gamma}(\varphi(\gamma)-\varphi(\gamma+1))^{2} \leq 4 \beta_{0}
$$

Finally, we give a proof for (3). By differentiating both sides of (3.2) w.r.t. $\omega_{\gamma}$, we have

$$
\begin{aligned}
\partial_{\omega_{\gamma}}^{2} E(\omega) & =2\left\langle\partial_{\omega_{\gamma}}\left(\Pi_{\gamma} \varphi\right), \varphi\right\rangle+2\left\langle\Pi_{\gamma} \varphi, \partial_{\omega_{\gamma}} \varphi\right\rangle \\
& =2\left\langle\Pi_{\gamma} \partial_{\omega_{\gamma}} \varphi, \varphi\right\rangle+2\left\langle\Pi_{\gamma} \varphi, \partial_{\omega_{\gamma}} \varphi\right\rangle=4\left\langle\Pi_{\gamma} \varphi, \partial_{\omega_{\gamma}} \varphi\right\rangle
\end{aligned}
$$

Next, we will compute $\partial_{\omega_{\gamma}} \varphi$.

Differentiating both sides of (3.1) with respect to $\omega_{\gamma}$ to get

$$
\begin{aligned}
\left(\partial_{\omega_{\gamma}} H_{\omega}(\Lambda)\right) \varphi+H_{\omega}(\Lambda) \partial_{\omega_{\gamma}} \varphi & =\partial_{\omega_{\gamma}} E(\omega) \varphi+E(\omega) \partial_{\omega_{\gamma}} \varphi \\
& =2\left\langle\Pi_{\gamma} \varphi, \varphi\right\rangle \varphi+E(\omega) \partial_{\omega_{\gamma}} \varphi
\end{aligned}
$$

Therefore,

$\left[H_{\omega}(\Lambda)-E(\omega)\right] \partial_{\omega_{\gamma}} \varphi=2\left\langle\Pi_{\gamma} \varphi, \varphi\right\rangle \varphi-\left(\partial_{\omega_{\gamma}} H_{\omega}(\Lambda)\right) \varphi=2\left(\left\langle\Pi_{\gamma} \varphi, \varphi\right\rangle \varphi-\Pi_{\gamma} \varphi\right)$.

Observe that $\psi_{\gamma}:=\left\langle\Pi_{\gamma} \varphi, \varphi\right\rangle \varphi-\Pi_{\gamma} \varphi \in\langle\varphi\rangle^{\perp}$, and $\left[H_{\omega}(\Lambda)-E(\omega)\right]$ is invertible in the subspace $\langle\varphi\rangle^{\perp}$ of $l^{2}(\Lambda)$, we get

$$
\partial_{\omega_{\gamma}} \varphi=2\left(H_{\omega}(\Lambda)-E(\omega)\right)^{-1}\left(\left\langle\Pi_{\gamma} \varphi, \varphi\right\rangle \varphi-\Pi_{\gamma} \varphi\right) .
$$

From (3.5) and (3.6), we obtain

$$
\partial_{\omega_{\gamma}}^{2} E(\omega)=4\left\langle\Pi_{\gamma} \varphi,\left(H_{\omega}(\Lambda)-E(\omega)\right)^{-1}\left(\left\langle\Pi_{\gamma} \varphi, \varphi\right\rangle \varphi-\Pi_{\gamma} \varphi\right)\right\rangle .
$$

Thanks to (3.6), we have $2\left(H_{\omega}(\Lambda)-E(\omega)\right)^{-1}\left(\left\langle\Pi_{\gamma} \varphi, \varphi\right\rangle \varphi-\Pi_{\gamma} \varphi\right)$ is orthogonal to $\varphi$. We therefore infer that

$$
\partial_{\omega_{\gamma}}^{2} E(\omega)=-4\left\langle\left(H_{\omega}(\Lambda)-E(\omega)\right)^{-1} \psi_{\gamma}, \psi_{\gamma}\right\rangle .
$$

Repeating this argument, it is not hard to prove that

$$
\partial_{\omega_{\gamma} \omega_{\beta}}^{2} E(\omega)=-4\left\langle\left(H_{\omega}(\Lambda)-E(\omega)\right)^{-1} \psi_{\gamma}, \psi_{\beta}\right\rangle
$$

for all $\gamma, \beta$. So, we have Lemma 3.1 proved.

Next, assume that $E(\omega)$ is an eigenvalue of $H_{\omega}(\Lambda)$ with $\Lambda=[-L, L]$. Recall that $\omega_{j} \in\left[\alpha_{0}, \beta_{0}\right]$ for all $j \in \mathbb{Z}$. From Lemma 3.1, we have $E(\omega) \in\left[0,4 \beta_{0}\right]$. Denote by $u:=u(\omega)$ the normalized eigenvector associated to $E(\omega)$. We would like to prove a "lower bound" for $u$ in the sense that there exists a large subset $J$ in $\Lambda$ such that the components $(u(k))_{k \in J}$ of $u$ can not be too small. 
Lemma 3.2. Pick $\beta \in(1 / 2,1)$. Then, there exists a point $k_{0}$ in $\Lambda$ and $a$ positive constant $\kappa$ depending only on $\alpha_{0}, \beta_{0}$ such that

$$
u^{2}(k)+u^{2}(k+1) \geqslant e^{-L^{\beta} / 2}
$$

for all $\left|k-k_{0}\right| \leqslant \kappa L^{\beta}$ when $L$ is large enough.

Proof of Lemma 3.2. We rewrite the eigenequation corresponding to the eigenvector $u$ and eigenvalue $E(\omega)$ at the point $n$ by means of the transfer matrix

$$
\left(\begin{array}{c}
u(n+1) \\
u(n)
\end{array}\right)=\left(\begin{array}{cc}
\frac{\omega_{n}+\omega_{n-1}-E(\omega)}{\omega_{n}} & \frac{-\omega_{n-1}}{\omega_{n}} \\
1 & 0
\end{array}\right)\left(\begin{array}{c}
u(n) \\
u(n-1)
\end{array}\right) .
$$

Now, let $T(n, E(\omega))$ and $v(n)$ denote the transfer matrix

$$
\left(\begin{array}{cc}
\frac{\omega_{n}+\omega_{n-1}-E(\omega)}{\omega_{n}} & \frac{-\omega_{n-1}}{\omega_{n}} \\
1 & 0
\end{array}\right)
$$

and the column vector $(u(n+1), u(n))^{t}$ respectively.

Then, for all $n$ greater than $m$ we have

$$
v(n)=T(n, E(\omega)) \cdots T(n-m+1, E(\omega)) v(m) .
$$

It is easy to check that the transfer matrices are invertible. Moreover, since $E(\omega) \in\left[0,4 \beta_{0}\right]$ and $0<\alpha_{0} \leq \omega_{j} \leq \beta_{0}$, they and their inverse matrices are uniformly bounded by a constant $C>1$ depending only on $\alpha_{0}$ and $\beta_{0}$.

Thus,

$$
\|v(n)\| \leqslant C^{|n-m|}\|v(m)\|=e^{(\log C)|n-m|}\|v(m)\|=e^{\eta|n-m|}\|v(m)\|
$$

for all $n, m$ in $\Lambda$ with $\eta=\log C>0$.

Assume that $\left\|v\left(k_{0}\right)\right\|$ is the maximum of $\|v(n)\|$. Hence,

$$
\left\|v\left(k_{0}\right)\right\| \geqslant \frac{1}{\sqrt{L}}
$$

from the fact that $\sum_{j \in \Lambda}\|v(j)\|^{2}=2$. Thus, for any $\kappa>0$, the following holds true

$$
\|v(k)\| \geqslant \frac{1}{\sqrt{L}} e^{-\eta\left|k-k_{0}\right|} \geq e^{-2 \kappa \eta L^{\beta}}
$$

for $\left|k-k_{0}\right| \leqslant \kappa L^{\beta}$ and $L$ sufficiently large.

In other words, we have

$$
u^{2}(k)+u^{2}(k+1) \geqslant e^{-4 \kappa \eta L^{\beta}}
$$

for all $\left|k-k_{0}\right| \leqslant \kappa L^{\beta}$. So, by choosing $\kappa=\frac{1}{8 \eta}$, we have Lemma 3.2 proved.

The following lemma is the main ingredient of the proof of the decorrelation estimate as well as the heart of the present paper: 
Lemma 3.3. Let $E \neq E^{\prime}$ be two positive energies in the localized regime and $\beta \in(1 / 2,1)$. Assume that $\Lambda=\Lambda_{L}=:[-L, L]$ is a large interval in $\mathbb{Z}$. Pick $c_{1}, c_{2}>0$ and denote by $\mathbb{P}^{*}$ the probability of the following event (called $(*))$ :

There exists two simple eigenvalues of $H_{\omega}(\Lambda)$, say $E(\omega), E^{\prime}(\omega)$ such that $|E(\omega)-E|+\left|E^{\prime}(\omega)-E^{\prime}\right| \leqslant e^{-L^{\beta}}$ and

$$
\left\|\nabla_{\omega}\left(c_{1} E(\omega)-c_{2} E^{\prime}(\omega)\right)\right\|_{1} \leqslant c_{1} e^{-L^{\beta}} .
$$

Then, there exists $c>0$ such that

$$
\mathbb{P}^{*} \leqslant e^{-c L^{2 \beta}} .
$$

Remark 3.4. There is a slightly difference between the above lemma and Lemma 2.4 in [8] where $c_{1}=c_{2}=1$. In fact, in the proof of Theorem 1.2, we will use the above lemma with $c_{1}, c_{2}$ are respectively $\frac{1}{E}, \frac{1}{E^{\prime}}$ which are two distinct, positive numbers. This difference results from the lack of the normalization of $\|\nabla E(\omega)\|_{1}$ for our model. Moreover, we will see in Remark 3.12 at the end of this section that, for the model (1.1), if $c_{1}=c_{2}, P^{*}$ is equal to 0 for all $L$ large.

We will skip for a moment the proof of Lemma 3.3 and recall how to use the above lemma to complete the proof of Theorem 1.2. This part can be found in [8]. We repeat it here with tiny but necessary changes adapted for the model (1.1).

Proof of Theorem 1.2. Recall that $E, E^{\prime}$ are two positive, fixed energies and $J_{L}=E+L^{-1}[-1,1]$ and $J_{L}^{\prime}=E^{\prime}+L^{-1}[-1,1]$. One chooses $L$ large enough such that $\min \left\{E-L^{-1}, E^{\prime}-L^{-1}\right\} \geq \min \left\{E, E^{\prime}\right\} / 2>0$.

Let $c L^{\alpha} \leqslant l \leqslant L^{\alpha} / c$ with $c>0$. From the Minami estimate, one has

$$
\begin{aligned}
& \mathbb{P}\left(\#\left\{\sigma\left(H_{\omega}\left(\Lambda_{l}\right)\right) \cap J_{L}\right\} \geqslant 2 \text { or } \#\left\{\sigma\left(H_{\omega}\left(\Lambda_{l}\right)\right) \cap J_{L}^{\prime}\right\} \geqslant 2\right) \\
& \leqslant \frac{4 \beta_{0}\|\rho\|_{\infty}\|s \rho(s)\|_{\infty}}{\left(\min \left\{E, E^{\prime}\right\}\right)^{2}}\left(\left|\Lambda_{l} \| J_{L}\right|\right)^{2} \leq C(l / L)^{2}
\end{aligned}
$$

where $C$ is a constant depending only on $E, E^{\prime}, \beta_{0}$ and $\rho$.

Hence, it is sufficient to prove that $\mathbb{P}_{0} \leqslant C(l / L)^{2} e^{(\log L)^{\beta}}$ where

$$
\mathbb{P}_{0}:=\mathbb{P}\left(\#\left\{\sigma\left(H_{\omega}\left(\Lambda_{l}\right)\right) \cap J_{L}\right\}=1 ; \#\left\{\sigma\left(H_{\omega}\left(\Lambda_{l}\right)\right) \cap J_{L}^{\prime}\right\}=1\right) .
$$

The crucial idea of proving decorrelation estimates in [8] is to reduce the proof of (3.7) to the proof of a similar estimate where $\Lambda_{l}$ is replaced by a much smaller cube, a cube of side length of order $\log L$. Precisely, one has (c.f. Lemma 2.1 and Lemma 2.2 in [8]):

$$
\mathbb{P}_{0} \leqslant C(l / L)^{2}+C(l / \widetilde{l}) \mathbb{P}_{1}
$$

where $\widetilde{l}=C \log L$ and

$$
\mathbb{P}_{1}:=\mathbb{P}\left(\#\left[\sigma\left(H_{\omega}\left(\Lambda_{\tilde{l}}\right)\right) \cap \widetilde{J}_{L}\right] \geqslant 1 \text { and } \#\left[\sigma\left(H_{\omega}\left(\Lambda_{\tilde{l}}\right)\right) \cap \widetilde{J}_{L}^{\prime}\right] \geqslant 1\right)
$$


where $\widetilde{J}_{L}=E+L^{-1}(-2,2)$ and $\widetilde{J}_{L}^{\prime}=E^{\prime}+L^{-1}(-2,2)$.

To complete the proof of Theorem 1.2, one need show that

$$
\mathbb{P}_{1} \leqslant C(\widetilde{l} / L)^{2} e^{\widetilde{l}^{\beta}}
$$

Thanks to the Minami estimate and the following inequality (see Lemma 2.3 in [8] for a proof)

$$
\left\|\operatorname{Hess}_{\omega}(E(\omega))\right\|_{l^{\infty} \rightarrow l^{1}} \leqslant \frac{C}{\operatorname{dist}\left(E(\omega), \sigma\left(H_{\omega}(\Lambda)\right) \backslash E(\omega)\right)},
$$

one infers that

$$
\mathbb{P}\left(\left\{\begin{array}{l}
\sigma\left(H_{\omega}\left(\Lambda_{\widetilde{l}}\right)\right) \cap\left(\widetilde{J}_{L}\right)=\{E(\omega)\} \\
\left\|\operatorname{Hess}_{\omega}(E(\omega))\right\|_{l^{\infty} \rightarrow l^{1}} \geqslant \epsilon^{-1}
\end{array}\right\}\right) \leqslant C \epsilon \widetilde{l}^{2} L^{-1} .
$$

Hence, for $\epsilon \in\left(4 L^{-1}, 1\right)$, one has

$$
\mathbb{P}_{1} \leqslant C \epsilon \widetilde{l}^{2} L^{-1}+\mathbb{P}_{\epsilon}
$$

where $\mathbb{P}_{\epsilon}=\mathbb{P}\left(\Omega_{0}(\epsilon)\right)$ with

$$
\Omega_{0}(\epsilon):=\left\{\begin{array}{c}
\sigma\left(H_{\omega}\left(\Lambda_{\tilde{l}}\right)\right) \cap \widetilde{J}_{L}=\{E(\omega)\} \\
\{E(\omega)\}=\sigma\left(H_{\omega}\left(\Lambda_{\tilde{l}}\right)\right) \cap(E-C \epsilon, E+C \epsilon) \\
\sigma\left(H_{\omega}\left(\Lambda_{\tilde{l}}\right)\right) \cap \widetilde{J}_{L}^{\prime}=\left\{E^{\prime}(\omega)\right\} \\
\left\{E^{\prime}(\omega)\right\}=\sigma\left(H_{\omega}\left(\Lambda_{\tilde{l}}\right)\right) \cap\left(E^{\prime}-C \epsilon, E^{\prime}+C \epsilon\right)
\end{array}\right\} .
$$

Next, one puts $\lambda:=e^{-\widetilde{l}^{\beta}}$ and defines, for $\gamma, \gamma^{\prime} \in \Lambda_{\tilde{l}}$,

$$
\Omega_{0, \beta}^{\gamma, \gamma^{\prime}}(\epsilon):=\Omega_{0}(\epsilon) \cap\left\{\omega|| J_{\gamma, \gamma^{\prime}}\left(E(\omega), E^{\prime}(\omega)\right) \mid \geqslant \lambda\right\}
$$

where $J_{\gamma, \gamma^{\prime}}\left(E(\omega), E^{\prime}(\omega)\right)$ is the Jacobian of the mapping

$$
\left(\omega_{\gamma}, \omega_{\gamma^{\prime}}\right) \mapsto\left(E(\omega), E^{\prime}(\omega)\right) .
$$

On the one hand, $\mathbb{P}_{\epsilon}$ can be dominated as follows:

$$
\mathbb{P}_{\epsilon} \leqslant \sum_{\gamma \neq \gamma^{\prime}} \mathbb{P}\left(\Omega_{0, \beta}^{\gamma, \gamma^{\prime}}(\epsilon)\right)+\mathbb{P}_{r}
$$

where $\mathbb{P}_{r}$ is the probability of the following event

$$
\mathcal{D}:=\left\{\omega \in \Omega_{0}(\epsilon)|| J_{\gamma, \gamma^{\prime}}\left(E(\omega), E^{\prime}(\omega)\right) \mid \leqslant \lambda \text { for all } \gamma, \gamma^{\prime} \in \Lambda_{\widetilde{l}}\right\} .
$$

On the other hand, from Lemma 2.6 in [8], it is known that

$$
\mathbb{P}\left(\Omega_{0, \beta}^{\gamma, \gamma^{\prime}}(\epsilon)\right) \leqslant C L^{-2} \lambda^{-4} \text { for all } \gamma, \gamma^{\prime} \in \Lambda_{\tilde{l}} .
$$

Hence,

$$
\mathbb{P}_{\epsilon} \leqslant C \widetilde{l}^{2} L^{-2} \lambda^{-4}+\mathbb{P}_{r}
$$

Choose $\epsilon:=L^{-1} \lambda^{-3},(3.10)$ and (3.11) yield that

$$
\mathbb{P}_{1} \leqslant C(\widetilde{l} / L)^{2} e^{\widetilde{l}^{\beta}}+\mathbb{P}_{r}
$$


Finally, we will use Lemma 3.3 to estimate $\mathbb{P}_{r}$.

For each $\omega \in \mathcal{D}$, we rewrite the Jacobian $J_{\gamma, \gamma^{\prime}}\left(E(\omega), E^{\prime}(\omega)\right)$ as follows:

$$
\begin{aligned}
& J_{\gamma, \gamma^{\prime}}\left(E(\omega), E^{\prime}(\omega)\right)=\left|\begin{array}{ll}
\partial_{\omega_{\gamma}} E(\omega) & \partial_{\omega_{\gamma^{\prime}}} E(\omega) \\
\partial_{\omega_{\gamma}} E^{\prime}(\omega) & \partial_{\omega_{\gamma^{\prime}}} E^{\prime}(\omega)
\end{array}\right| \\
& =\frac{E(\omega) E^{\prime}(\omega)}{\omega_{\gamma} \omega_{\gamma^{\prime}}}\left|\begin{array}{cc}
\frac{1}{E(\omega)} \omega_{\gamma} \partial_{\omega_{\gamma}} E(\omega) & \frac{1}{E(\omega)} \omega_{\gamma^{\prime}} \partial_{\omega_{\gamma^{\prime}}} E(\omega) \\
\frac{1}{E^{\prime}(\omega)} \omega_{\gamma} \partial_{\omega_{\gamma}} E^{\prime}(\omega) & \frac{1}{E^{\prime}(\omega)} \omega_{\gamma^{\prime}} \partial_{\omega_{\gamma^{\prime}}} E^{\prime}(\omega)
\end{array}\right|
\end{aligned}
$$

if $E(\omega)$ and $E^{\prime}(\omega)$ are non-zero.

Note that, from (3.3), one has

$$
\sum_{\gamma \in \Lambda_{\tilde{l}}} \frac{1}{E(\omega)} \omega_{\gamma} \partial_{\omega_{\gamma}} E(\omega)=\sum_{\gamma \in \Lambda_{\tilde{l}}} \frac{1}{E^{\prime}(\omega)} \omega_{\gamma} \partial_{\omega_{\gamma}} E^{\prime}(\omega)=1 .
$$

Hence, one can apply Lemma 2.5 in [8] to (3.13) and deduce that

$$
\left\|\nabla_{\omega}\left(\frac{1}{E} E(\omega)-\frac{1}{E^{\prime}} E^{\prime}(\omega)\right)\right\|_{1} \leqslant e^{-\widetilde{l}^{\beta^{\prime}}}
$$

for any $1 / 2<\beta^{\prime}<\beta$.

Thus, Lemma 3.3 yields that, for $L$ sufficiently large,

$$
\mathbb{P}_{r} \leqslant \widetilde{l}^{2} e^{-\widetilde{l^{2} \beta^{\prime}}}=O\left(L^{-\infty}\right) .
$$

From (3.12) and (3.14), (3.8) follows and we have Theorem 1.2 proved.

Before coming to the proof of Lemma 3.3, we state and prove here a short lemma which will be used repeatedly in the rest of this section.

Lemma 3.5. Pick $A \in \operatorname{Mat}_{n}(\mathbb{R})$ and $b \in \mathbb{R}^{n}$ such that $\|b\| \leq c_{0} e^{-L^{\beta} / 2}$ where $c_{0}, L$ are fixed, positive constants. Assume that the following system of linear equations

$$
\begin{array}{r}
A x=b \\
\text { has a solution } u \text { satisfying }\|u\| \geq e^{-L^{\beta} / 4} .
\end{array}
$$

Then,

$$
|\operatorname{det} A| \leq c_{0} \max \{1,\|\operatorname{adj}(A)\|\} e^{-L^{\beta} / 4}
$$

where $\operatorname{adj}(A)$ is the adjugate of the matrix $A$.

Proof of Lemma 3.5. Assume by contradiction that

$$
|\operatorname{det} A|>c_{0} \max \{1,\|\operatorname{adj}(A)\|\} e^{-L^{\beta} / 4}>0 .
$$

As a result, $A$ is invertible. Hence, $u=A^{-1} b$ is the unique solution of the system $A x=b$. We therefore infer that

$$
\|u\| \leq\left\|A^{-1}\right\|\|b\|=\frac{1}{|\operatorname{det} A|}\|\operatorname{adj}(A)\|\|b\| \leq \frac{\max \{1,\|\operatorname{adj}(A)\|\}}{|\operatorname{det} A|}\|b\| .
$$

Hence,

$$
|\operatorname{det} A| \leq c_{0} \max \{1,\|\operatorname{adj}(A)\|\} e^{-L^{\beta} / 2} e^{L^{\beta} / 4}=c_{0} \max \{1,\|\operatorname{adj}(A)\|\} e^{-L^{\beta} / 4}
$$


which is a contradiction.

To complete the present section, we state here the proof of Lemma 3.3.

Proof of Lemma 3.3. Let $u:=u(\omega)$ and $v:=v(\omega)$ be normalized eigenvectors associated to $E(\omega)$ and $E^{\prime}(\omega)$. By Lemma 3.1, we have

$$
\nabla_{\omega} E(\omega)=\left(2\left\|\Pi_{\gamma} u\right\|^{2}\right)_{\gamma \in \Lambda} \text { and } \nabla_{\omega} E^{\prime}(\omega)=\left(2\left\|\Pi_{\gamma} v\right\|^{2}\right)_{\gamma \in \Lambda}
$$

We introduce the linear operator $T$ from $l^{2}(\Lambda)$ to $l^{2}(\Lambda)$ defined as follows

$$
T u(n)=u(n)-u(n+1)
$$

where $u=(u(n))_{n} \in l^{2}(\Lambda)$. Recall that $\Lambda=\Lambda_{L}=\mathbb{Z} / L \mathbb{Z}$, i.e. we use periodic boundary conditions here.

Assume that $\{\omega\}_{j \in \Lambda}$ belongs to the event $(*)$. We thus have

$$
\begin{aligned}
c_{1} e^{-L^{\beta}} & \geqslant\left\|\nabla_{\omega}\left(c_{1} E(\omega)-c_{2} E^{\prime}(\omega)\right)\right\|_{1}=\sum_{n}\left|\left(\sqrt{c_{1}} T u(n)\right)^{2}-\left(\sqrt{c_{2}} T v(n)\right)^{2}\right| \\
& =\sum_{n}\left|\sqrt{c_{1}} T u(n)-\sqrt{c_{2}} T v(n) \| \sqrt{c_{1}} T u(n)+\sqrt{c_{2}} T v(n)\right| .
\end{aligned}
$$

Hence, $e^{-L^{\beta}} \geqslant \sum_{n}|T u(n)-c T v(n)||T u(n)+c T v(n)|$ with $c=\sqrt{c_{2}} / \sqrt{c_{1}}>0$. Then, there exists a partition of $\Lambda=\{-L, \ldots, L\}$, say $\mathcal{P} \subset \Lambda$ and $\mathcal{Q} \subset \Lambda$ such that $\mathcal{P} \cup \mathcal{Q}=\Lambda, \mathcal{P} \cap \mathcal{Q}=\emptyset$ and

- for $n \in \mathcal{P},|T u(n)-c T v(n)| \leqslant e^{-L^{\beta} / 2}$

- for $n \in \mathcal{Q},|T u(n)+c T v(n)| \leqslant e^{-L^{\beta} / 2}$

From now on, we put $v(n):=c v(n)$. This abuse of notation changes nothing thanks to the linearity of the operator $T$.

Hence, we obtain that

$$
\begin{cases}\mid T u(n)=T v(n)+\mathrm{O}\left(e^{-L^{\beta} / 2}\right) & \text { if } n \in \mathcal{P} \\ \mid T u(n)=-T v(n)+\mathrm{O}\left(e^{-L^{\beta} / 2}\right) & \text { if } n \in \mathcal{Q}\end{cases}
$$

From Lemma 3.2, there exists $c_{3}>0$ depending only on $\alpha_{0}, \beta_{0}$ and an interval $J$ of the length $c_{3} L^{\beta}$ such that

$$
|u(k)|^{2}+|u(k+1)|^{2} \geqslant 2 e^{-L^{\beta} / 2}
$$

for all $k \in J$.

Now, we decompose

$$
\mathcal{P} \cap J=\cup \mathcal{P}_{j} \text { and } \mathcal{Q} \cap J=\cup \mathcal{Q}_{j}
$$

where $\mathcal{P}_{j}$ and $\mathcal{Q}_{j}$ are intervals in $\mathbb{Z}$.

We will divide the rest of the proof into some lemmata. First of all, in the Lemma 3.6, we show a restriction on the length of each interval $\mathcal{P}_{j}$ and $\mathcal{Q}_{j}$ in $\mathbb{Z}$. We will make use of this lemma later to prove a "reduction" lemma (Lemma 3.10). Next, In Lemma 3.8, with any four consecutive points in $J$, we explain how to form a (inhomogeneous) $10 \times 10$ system of linear equations from (3.15) and eigenequations for $u$ and $v$. Finally, we show some 
restrictions on $\left\{\omega_{j}\right\}_{j \in \Lambda}$ in Lemma 3.11. Thanks to this lemma and Lemma 3.10, Lemma 3.3 follows.

Lemma 3.6. Assume that $\left\{\omega_{j}\right\}_{j \in \Lambda}$ belongs to the event $\left(^{*}\right)$ defined in Lemma 3.3: $H_{\omega}(\Lambda)$ has two simple eigenvalues $E(\omega), E^{\prime}(\omega)$ such that $\mid E(\omega)-$ $E|+| E^{\prime}(\omega)-E^{\prime} \mid \leqslant e^{-L^{\beta}}$ and

$$
\left\|\nabla_{\omega}\left(c_{1} E(\omega)-c_{2} E^{\prime}(\omega)\right)\right\|_{1} \leqslant c_{1} e^{-L^{\beta}} .
$$

Denote by $u, v$ normalized eigenvectors associated to $E(\omega), E^{\prime}(\omega)$ respectively and consider the decomposition $\left\{\mathcal{P}_{i}, \mathcal{Q}_{j}\right\}$ in (3.17). Then, any $\mathcal{P}_{j}$ or $\mathcal{Q}_{j}$ can not contain more than four points.

Proof of Lemma 3.6. Thanks to the equivalent role of $\mathcal{P}$ and $\mathcal{Q}$, it is sufficient to prove Lemma 3.6 for $\left\{\mathcal{P}_{j}\right\}_{j}$.

Assume by contradiction that there exists an interval $\mathcal{P}_{j}$ contain at least five consecutive points, say $\mathcal{P}_{j}=\{n-2, n-1, n, n+1, n+2, \ldots, m\}$ with $m \geqslant n+2$.

First of all, thanks to (3.15), we have

$$
\begin{aligned}
T u(n-2) & =T v(n-2)+O\left(e^{-L^{\beta} / 2}\right) . \\
T u(n-1) & =T v(n-1)+O\left(e^{-L^{\beta} / 2}\right) . \\
T u(n) & =T v(n)+O\left(e^{-L^{\beta} / 2}\right) . \\
T u(n+1) & =T v(n+1)+O\left(e^{-L^{\beta} / 2}\right) . \\
T u(n+2) & =T v(n+2)+O\left(e^{-L^{\beta} / 2}\right) .
\end{aligned}
$$

Next, consider the triple of consecutive points $\{n-2, n-1, n\} \in \mathcal{P}_{j}$. Using the eigenequations for $u$ and $v$ at the point $(n-1)$ and take the hypothesis $|E(\omega)-E|+\left|E^{\prime}(\omega)-E^{\prime}\right| \leq e^{-L^{\beta}}$ into account, we deduce

$$
\begin{aligned}
& E u(n-1)=\omega_{n-1} T u(n-1)-\omega_{n-2} T u(n-2)+O\left(e^{-L^{\beta} / 2}\right), \\
& E^{\prime} v(n-1)=\omega_{n-1} T v(n-1)-\omega_{n-2} T v(n-2)+O\left(e^{-L^{\beta} / 2}\right) .
\end{aligned}
$$

Hence, (3.19), (3.20) and the first two equations in (3.18) yield

$$
E u(n-1)=E^{\prime} v(n-1)+O\left(e^{-L^{\beta} / 2}\right) .
$$

Similarly, we have

$$
E u(n)=E^{\prime} v(n)+O\left(e^{-L^{\beta} / 2}\right) .
$$

Combining (3.21), (3.22) and the second equation in (3.18), we obtain

$$
\left(1-\frac{E}{E^{\prime}}\right) u(n)=\left(1-\frac{E}{E^{\prime}}\right) u(n-1)+O\left(e^{-L^{\beta} / 2}\right)
$$

which implies that

$$
T u(n-1) \leq C e^{-L^{\beta} / 2}
$$


where $C$ is a positive constant depending only on $E, E^{\prime}, \alpha_{0}$ and $\beta_{0}$. Repeating again the above argument for other triples of consecutive points in $\mathcal{P}_{j}$, we obtain

$$
T u(n) \leq C e^{-L^{\beta} / 2},
$$

and

$$
T u(n+1) \leq C e^{-L^{\beta} / 2} .
$$

On the other hand, we have the following eigenequations for $u$ at the point $n$ and $n+1$

$$
\begin{aligned}
E u(n) & =\omega_{n} T u(n)-\omega_{n-1} T u(n-1)+O\left(e^{-L^{\beta} / 2}\right), \\
E u(n+1) & =\omega_{n+1} T u(n+1)-\omega_{n} T u(n)+O\left(e^{-L^{\beta} / 2}\right) .
\end{aligned}
$$

Hence, combining the above equations and (3.23)-(3.25), we infer that there exists a positive constant $C$ being independent of $L$ such that

$$
|u(n)|^{2}+|u(n+1)|^{2} \leqslant C e^{-L^{\beta}}
$$

which contradicts (3.16) if we choose $L$ large enough. Hence, an interval $\mathcal{P}_{j}$ or $\mathcal{Q}_{j}$ can not contain more than four points in $\mathbb{Z}$ and we have Lemma 3.6 proved.

From the proof of Lemma 3.6, we reach to the following conclusion:

Remark 3.7. If two consecutive points ordered increasingly belong to some interval $\mathcal{P}_{j}(n-2, n-1$ for instance), the value of $u$ at the latter point $(n-1$ in this case) is proportional to the value of $v$ at that point (as in (3.21)) up to an exponentially small error. Moreover, if we have three consecutive points ordered increasingly in some interval $\mathcal{P}_{j}(n-2, n-1, n)$, the middle point $(n-1)$ always satisfies an inequality of the form (3.23). Finally, if three points $n-2, n-1, n$ belong to some $\mathcal{Q}_{i}$, we will have almost the same conclusion except that $E^{\prime}$ need replacing by $-E^{\prime}$ in (3.21) and (3.22).

Lemma 3.8. Let $J$ be the subinterval of $\Lambda$ where (3.16) holds and $n-2, n-$ $1, n, n+1$ be four consecutive points in $J$. Assume the same hypotheses as in Lemma 3.6 and put $U:=(u(n-2), \ldots, u(n+2), v(n-2), \ldots, v(n+2))^{t}$. Then, from (3.15) and eigenequations for $u$ and $v$, we can form a $10 \times 10$ system of linear equations which admits $U$ as one of its solutions.

Proof of Lemma 3.8. From (3.15), for each of these four points, we have an equation of the form

$$
T u(k)= \pm T v(k)+O\left(e^{-L^{\beta} / 2}\right), k=\overline{n-2, n+1}
$$

where the choice of $(+)$ or $(-)$ sign depends on whether $k$ belongs to $\mathcal{P}$ or $\mathcal{Q}$. So, we have 4 (inhomogeneous) linear equations in hand.

On the other hand, we have 6 eigenequations of eigenvectors $u$ and $v$ at the points $n-1, n$ and $n+1$. Hence, apparently, we have 10 linear equations 
corresponding to 10 variables $\{u(n-2), \ldots, u(n+2), v(n-2), \ldots, v(n+2)\}$. However, there is a couple of things here which should be made clearer.

First of all, to form our systems of linear equations, we use the following three eigenequations w.r.t. $u$

$$
E u(k)=\omega_{k} T u(k)-\omega_{k-1} T u(k-1)+O\left(e^{-L^{\beta} / 2}\right)
$$

where $k=\overline{n-1, n+1}$.

Next, we consider the eigenequations of $v$ at $k=\overline{n-1, n+1}$

$$
E^{\prime} v(k)=\omega_{k} T v(k)-\omega_{k-1} T v(k-1)+O\left(e^{-L^{\beta} / 2}\right) .
$$

Instead of using directly (3.28) for our $10 \times 10$ systems of linear equations, we substitute (3.26) into the right hand side of (3.28) to get

$$
E^{\prime} v(k)= \pm \omega_{k} T u(k) \mp \omega_{k-1} T u(k-1)+O\left(e^{-L^{\beta} / 2}\right)
$$

and (3.29) will be used for our $10 \times 10$ systems of linear equations.

In Lemma 3.11, we will write down these $10 \times 10$ systems of linear equations as follows: The first four equations come from (3.26). Then, we write down the equations in (3.27) and (3.29). The fifth equation is (3.27) and the sixth is (3.29) with $k=n$. The seventh is (3.27) and the eighth is (3.29) with $k=n+1$. Lastly, (3.27) and (3.29) with $k=n-1$ are the ninth and the tenth equation in turn.

Finally, we make an important remark in case there exists an interval $\mathcal{P}_{j}$ or $\mathcal{Q}_{j}$ contains at least two consecutive points of these four points, says $j-1, j$. According to Remark 3.7, we have

$$
v(j)= \pm \frac{E}{E^{\prime}} u(j)+O\left(e^{-L^{\beta} / 2}\right)
$$

where (3.30) takes $(+)$ sign iff $j-1$ and $j \in \mathcal{P}$.

Whenever (3.30) holds true, we will use it to replace (3.29) w.r.t. $k=j$ in our systems of linear equations. This replacement simplifies these $10 \times 10$ systems of linear equations and makes them easier to analyze.

Definition 3.9. A point $n \in J$ is an interior point of $J$ if the interval $[n-2, n+2]$ belongs to $J$.

Let $n-2, n-1, n, n+1$ be interior points in $J$. We consider all possible $10 \times 10$ systems of linear equations which we can get from these points as in Lemma 3.8. We have four points, each of them can belong to $\mathcal{P}$ or $\mathcal{Q}$. Hence, the number of choices for four points' belonging to $\mathcal{P}$ or $\mathcal{Q}$ equals $2^{4}=16$ which is also the total number of $10 \times 10$ systems of linear equations obtained in Lemma 3.8. Furthermore, we have the following useful observation:

Lemma 3.10. Assume the same hypotheses as in Lemma 3.6. Let $n-$ $2, n-1, n, n+1$ be interior points in $J$ and $\left\{\mathcal{P}_{i}, \mathcal{Q}_{j}\right\}$ be the decomposition in (3.17). Then, we will only need to analyze $10 \times 10$ systems of linear equations corresponding to the following four cases: 

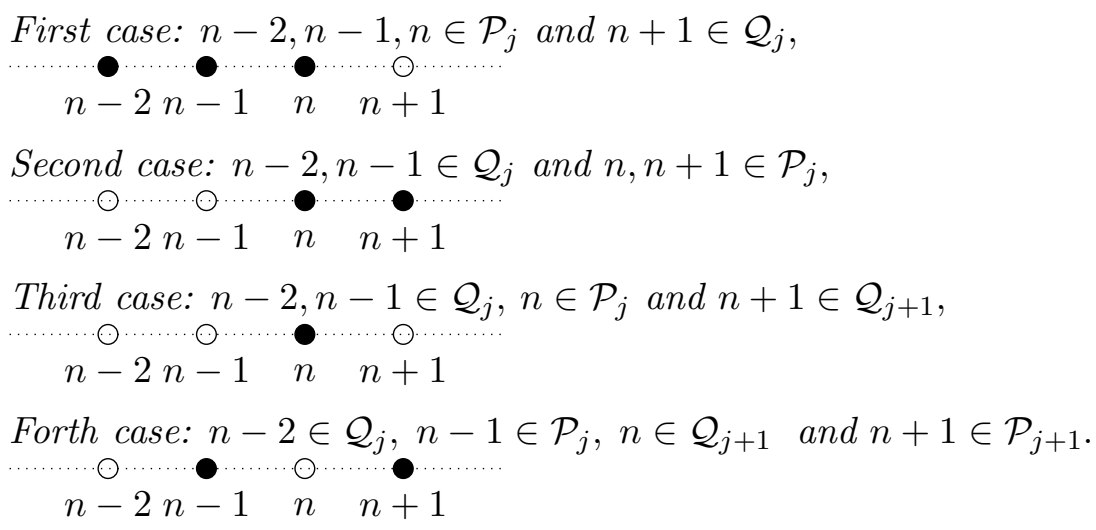

Proof of Lemma 3.10. As mentioned above, we have a total of 16 systems of linear equations to analyze. Thanks to the equivalent role of $\mathcal{P}$ and $\mathcal{Q}$, we only have to consider a half of them. Apart from four cases listed above, the other cases could be:

Fifth case: Assume that all of these four points belong to some $\mathcal{P}_{j}$.

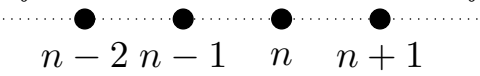

Hence, From Lemma 3.6, $n+2 \in \mathcal{Q}_{j}$. We consider four points $n-1, n, n+$ $1, n+2$ and come back to First case.

Sixth case: Suppose that $n-2 \in \mathcal{Q}_{j}, n-1, n \in \mathcal{P}_{j}$, and $n+1 \in \mathcal{Q}_{j+1}$.

$$
n-2 n-1 \quad n \quad n+1
$$

We consider the point $n+2$. If $n+2$ belongs to $\mathcal{Q}_{j+1}$, we consider four points $n-1, n, n+1, n+2$ and come back to Second case because of the equivalent role of $\mathcal{P}$ and $\mathcal{Q}$. Otherwise, $n+2$ belongs to $\mathcal{P}_{j+1}$. In this case, we consider four points $n-1, n, n+1, n+2$ and come back to Third case.

Seventh case: Assume that $n-2 \in \mathcal{Q}_{j}, n-1 \in \mathcal{P}_{j}$ and $n, n+1 \in \mathcal{Q}_{j+1}$.

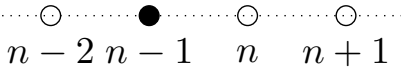

In this case, we consider four points $n-3, n-2, n-1, n$. If $n-3$ also belongs to $\mathcal{Q}_{j}$, we come back to Third case. Otherwise, we come back to Forth case on account of the equivalent role of $\mathcal{P}$ and $\mathcal{Q}$.

Eighth case: Suppose that $n-2 \in Q_{j}$ and $n-1, n, n+1 \in \mathcal{P}_{j}$ for some $j$.

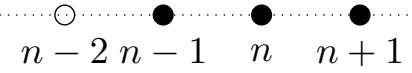

If $n+2 \in \mathcal{Q}_{j+1}$, we consider four points $n-1, n, n+1, n+2$ and come back to First case. Otherwise, $n+2$ still belongs to $\mathcal{P}_{j}$, hence $n+3 \in \mathcal{Q}_{j+1}$ according to Lemma 3.6. On the other hand, $n+3$ still belongs to $J$ since $n+1$ is the interior point of $J$. Hence, we consider four points $n, n+1, n+2, n+3$ and come back to First case.

To conclude, we only need to analyze 4 special cases. The other cases can be reduced to those ones. 
Now, we come to the final stage in the proof of Lemma 3.3 where we deduce the restrictions on r.v.'s $\omega_{j}$.

Lemma 3.11. Assume hypotheses as in Lemma 3.6. Let $J$ be the interval defined in (3.15) and $n-2, n-1, n, n+1$ be four interior points of $J$. Assume that these four points correspond to one of the four cases listed in Lemma 3.10. Then, one of the following restrictions on r.v.'s holds true

$$
\begin{gathered}
\text { (i) }\left|\omega_{n}+\frac{E^{\prime}-E}{4}\right| \leqslant C e^{-L^{\beta} / 8}, \\
\text { (ii) }\left|\omega_{n-1}+\frac{E^{\prime}-E}{4}\right| \leqslant C e^{-L^{\beta} / 8}, \\
\text { (iii) }\left|\omega_{n-1} \omega_{n}-\frac{\left(E+E^{\prime}\right)^{2}}{4}\right| \leqslant C e^{-L^{\beta} / 4} .
\end{gathered}
$$

Proof of Lemma 3.11. For each of four cases in Lemma 3.10, we consider the corresponding system of linear equations formed in Lemma 3.8 and compute its determinant. This yields some restrictions on r.v.'s.

Recall that $U:=(u(n-2), \ldots, u(n+2), v(n-2), \ldots, v(n+2))^{t}$.

First case: Assume that three points $n-2, n-1, n \in \mathcal{P}_{j}$ and the other one $n+1 \in \mathcal{Q}_{j}$.

Since $n-2, n-1, n \in \mathcal{P}_{j}$, two equations in (3.29) associated to $n-1, n$ will be replaced by two equations of the type (3.30) with $(+)$ sign in our system. Hence, according to Lemma 3.8, $U$ satisfies the following system of linear equations:

$$
A_{0} U=b_{0}
$$

where $b_{0}=\left(b_{0}^{j}\right)_{1 \leqslant j \leqslant 10}$ with $\left\|b_{0}\right\| \leqslant e^{-L^{\beta} / 2}$ and $A_{0}$ is the $10 \times 10$ matrix of the block form $\left(A_{0}^{1} \mid A_{0}^{2}\right)$ with

$$
A_{0}^{1}:=\left(\begin{array}{ccccc}
1 & -1 & 0 & 0 & 0 \\
0 & 1 & -1 & 0 & 0 \\
0 & 0 & 1 & -1 & 0 \\
0 & 0 & 0 & 1 & -1 \\
0 & -\omega_{n-1} & \omega_{n-1}+\omega_{n}-E & -\omega_{n} & 0 \\
0 & 0 & \frac{E}{E^{\prime}} & 0 & 0 \\
0 & 0 & -\omega_{n} & \omega_{n}+\omega_{n+1}-E & -\omega_{n+1} \\
0 & 0 & \omega_{n} & \omega_{n+1}-\omega_{n} & -\omega_{n+1} \\
-\omega_{n-2} & \omega_{n-2}+\omega_{n-1}-E & -\omega_{n-1} & 0 & 0 \\
0 & \frac{E}{E^{\prime}} & 0 & 0 & 0
\end{array}\right)
$$


and

$$
A_{0}^{2}:=\left(\begin{array}{ccccc}
1 & -1 & 0 & 0 & 0 \\
0 & 1 & -1 & 0 & 0 \\
0 & 0 & 1 & -1 & 0 \\
0 & 0 & 0 & -1 & 1 \\
0 & 0 & 0 & 0 & 0 \\
0 & 0 & -1 & 0 & 0 \\
0 & 0 & 0 & 0 & 0 \\
0 & 0 & 0 & E^{\prime} & 0 \\
0 & 0 & 0 & 0 & 0 \\
0 & -1 & 0 & 0 & 0
\end{array}\right) .
$$

Let $\operatorname{adj}\left(A_{0}\right)$ be the adjugate of $A_{0}$. It is easy to see that

$$
\max \left\{1,\left\|\operatorname{adj}\left(A_{0}\right)\right\|\right\} \leqslant M_{0}
$$

where $M_{0}$ is a positive constant depending only on $E, E^{\prime}, \alpha_{0}, \beta_{0}$. Hence, thanks to Lemma 3.5, we have

$$
\left|\operatorname{det} A_{0}\right| \leq M_{0} e^{-L^{\beta} / 4}
$$

By an explicit computation in Appendix A, we have

$$
\left|\operatorname{det} A_{0}\right|=\frac{4 E}{E^{\prime}}\left(E+E^{\prime}\right) \omega_{n-2} \omega_{n+1}\left|\omega_{n}+\frac{E^{\prime}-E}{4}\right| \leqslant M_{0} e^{-L^{\beta} / 4} .
$$

Therefore, from the fact that $E, E^{\prime}>0$ and $\omega_{j} \geq \alpha_{0}>0$, the following condition on $\omega$ holds true with $L$ sufficiently large:

$$
\left|\omega_{n}+\frac{E^{\prime}-E}{4}\right| \leqslant C e^{-L^{\beta} / 4}
$$

Second case: $n-2, n-1 \in \mathcal{Q}_{j}$ and $n, n+1 \in \mathcal{P}_{j}$.

In the present case, since $n-2, n-1 \in \mathcal{Q}_{j}$, we use (3.30) with (-) sign w.r.t. $n-1$ to replace the equation in (3.29) w.r.t. $n-1$ for our system of linear equations. Besides, since $n, n+1 \in \mathcal{P}_{j}$, the equation in (3.29) w.r.t. $n+1$ will be replaced by (3.30) with $(+)$ sign at $n+1$.

Hence, according to Lemma 3.8, we have the following $10 \times 10$ system of linear equations:

$$
A_{1} U=b_{1}
$$


where $b_{1}=\left(b_{1}^{j}\right)_{1 \leqslant j \leqslant 10}$ with $\left\|b_{1}\right\| \leqslant 10 e^{-L^{\beta} / 2}$ and $A_{1}=\left(A_{1}^{1} \mid A_{1}^{2}\right)$ where

$$
A_{1}^{1}:=\left(\begin{array}{ccccc}
1 & -1 & 0 & 0 & 0 \\
0 & 1 & -1 & 0 & 0 \\
0 & 0 & 1 & -1 & 0 \\
0 & 0 & 0 & 1 & -1 \\
0 & -\omega_{n-1} & \omega_{n-1}+\omega_{n}-E & -\omega_{n} & 0 \\
0 & \omega_{n-1} & \omega_{n}-\omega_{n-1} & -\omega_{n} & 0 \\
0 & 0 & -\omega_{n} & \omega_{n}+\omega_{n+1}-E & -\omega_{n+1} \\
0 & 0 & 0 & \frac{E}{E^{\prime}} & 0 \\
-\omega_{n-2} & \omega_{n-2}+\omega_{n-1}-E & -\omega_{n-1} & 0 & 0 \\
0 & -\frac{E}{E^{\prime}} & 0 & 0 & 0
\end{array}\right)
$$

and

$$
A_{1}^{2}:=\left(\begin{array}{ccccc}
-1 & 1 & 0 & 0 & 0 \\
0 & -1 & 1 & 0 & 0 \\
0 & 0 & 1 & -1 & 0 \\
0 & 0 & 0 & 1 & -1 \\
0 & 0 & 0 & 0 & 0 \\
0 & 0 & -E^{\prime} & 0 & 0 \\
0 & 0 & 0 & 0 & 0 \\
0 & 0 & 0 & -1 & 0 \\
0 & 0 & 0 & 0 & 0 \\
0 & -1 & 0 & 0 & 0
\end{array}\right)
$$

Again, by using Lemma 3.5, we infer that

$$
\left|\operatorname{det} A_{1}\right| \leqslant M_{1} e^{-L^{\beta} / 4}
$$

where $M_{1}=M_{1}\left(E, E^{\prime}, \alpha_{0}, \beta_{0}\right)>0$.

Compute the determinant of $A_{1}$ (See Appendix A), we obtain

$$
\left|\operatorname{det} A_{1}\right|=\frac{4 E}{E^{\prime}} \omega_{n-2} \omega_{n+1}\left|\omega_{n-1} \omega_{n}-\frac{\left(E+E^{\prime}\right)^{2}}{4}\right| \leqslant M_{1} e^{-L^{\beta} / 4} .
$$

Hence, take $\omega_{j} \geqslant \alpha_{0}>0$ and $E, E^{\prime}>0$ into account, we have

$$
\left|\omega_{n-1} \omega_{n}-\frac{\left(E+E^{\prime}\right)^{2}}{4}\right| \leqslant C e^{-L^{\beta} / 4}
$$

as $L$ sufficiently large.

Third case: $n-2, n-1 \in \mathcal{Q}_{j}, n \in \mathcal{P}_{j}$ and $n+1 \in \mathcal{Q}_{j+1}$.

According to Step 2 and Lemma 3.5, we have

$$
A_{2} U=b_{2}
$$


where $A_{2}=\left(A_{2}^{1} \mid A_{2}^{2}\right)$ is the $10 \times 10$ matrix defined by

$$
A_{2}^{1}:=\left(\begin{array}{ccccc}
1 & -1 & 0 & 0 & 0 \\
0 & 1 & -1 & 0 & 0 \\
0 & 0 & 1 & -1 & 0 \\
0 & 0 & 0 & 1 & -1 \\
0 & -\omega_{n-1} & \omega_{n-1}+\omega_{n}-E & -\omega_{n} & 0 \\
0 & \omega_{n-1} & \omega_{n}-\omega_{n-1} & -\omega_{n} & 0 \\
0 & 0 & -\omega_{n} & \omega_{n}+\omega_{n+1}-E & -\omega_{n+1} \\
0 & 0 & -\omega_{n} & \omega_{n}-\omega_{n+1} & \omega_{n+1} \\
-\omega_{n-2} & \omega_{n-2}+\omega_{n-1}-E & -\omega_{n-1} & 0 & 0 \\
0 & -\frac{E}{E^{\prime}} & 0 & 0 & 0
\end{array}\right)
$$

and

$$
A_{2}^{2}:=\left(\begin{array}{ccccc}
-1 & 1 & 0 & 0 & 0 \\
0 & -1 & 1 & 0 & 0 \\
0 & 0 & 1 & -1 & 0 \\
0 & 0 & 0 & -1 & 1 \\
0 & 0 & 0 & 0 & 0 \\
0 & 0 & -E^{\prime} & 0 & 0 \\
0 & 0 & 0 & 0 & 0 \\
0 & 0 & 0 & -E^{\prime} & 0 \\
0 & 0 & 0 & 0 & 0 \\
0 & -1 & 0 & 0 & 0
\end{array}\right)
$$

such that $\left|\operatorname{det} A_{2}\right| \leq M_{2} e^{-L^{\beta} / 4}$ where $M_{2}$ is some positive constant. Then, by an explicit computation, we obtain that

$$
\left|\operatorname{det} A_{2}\right|=4 E\left(E+E^{\prime}\right) \omega_{n-2} \omega_{n+1}\left|\omega_{n}+\frac{E^{\prime}-E}{4}\right| \leqslant M_{2} e^{-L^{\beta} / 4}
$$

which immediately yields that

$$
\left|\omega_{n}+\frac{E^{\prime}-E}{4}\right| \leqslant C e^{-L^{\beta} / 4}
$$

as $L \rightarrow+\infty$.

Forth case: Suppose that $n-2 \in \mathcal{Q}_{j}, n-1 \in \mathcal{P}_{j}, n \in \mathcal{Q}_{j+1}$ and $n+1 \in \mathcal{P}_{j+1}$. In this case, $U$ satisfies the following system of linear equations:

$$
A_{3} U=b_{3}
$$


where $A_{3}=\left(A_{3}^{1} \mid A_{3}^{2}\right)$ is the $10 \times 10$ matrix defined by

$$
A_{3}^{1}:=\left(\begin{array}{ccccc}
1 & -1 & 0 & 0 & 0 \\
0 & 1 & -1 & 0 & 0 \\
0 & 0 & 1 & -1 & 0 \\
0 & 0 & 0 & 1 & -1 \\
0 & -\omega_{n-1} & \omega_{n-1}+\omega_{n}-E & -\omega_{n} & 0 \\
0 & -\omega_{n-1} & \omega_{n-1}-\omega_{n} & \omega_{n} & 0 \\
0 & 0 & -\omega_{n} & \omega_{n}+\omega_{n+1}-E & -\omega_{n+1} \\
0 & 0 & \omega_{n} & \omega_{n+1}-\omega_{n} & -\omega_{n+1} \\
-\omega_{n-2} & \omega_{n-2}+\omega_{n-1}-E & -\omega_{n-1} & 0 & 0 \\
\omega_{n-2} & \omega_{n-1}-\omega_{n-2} & -\omega_{n-1} & 0 & 0
\end{array}\right)
$$

and

$$
A_{3}^{2}:=\left(\begin{array}{ccccc}
-1 & 1 & 0 & 0 & 0 \\
0 & 1 & -1 & 0 & 0 \\
0 & 0 & -1 & 1 & 0 \\
0 & 0 & 0 & 1 & -1 \\
0 & 0 & 0 & 0 & 0 \\
0 & 0 & -E^{\prime} & 0 & 0 \\
0 & 0 & 0 & 0 & 0 \\
0 & 0 & 0 & -E^{\prime} & 0 \\
0 & 0 & 0 & 0 & 0 \\
0 & -E^{\prime} & 0 & 0 & 0
\end{array}\right)
$$

and $\left|\operatorname{det} A_{3}\right| \leq M_{3} e^{-L^{\beta} / 4}$.

We compute

$$
\left|\operatorname{det} A_{3}\right|=E E^{\prime} \omega_{n-2} \times \omega_{n+1} \times\left|4 \omega_{n-1}+E^{\prime}-E\right| \times\left|E^{\prime}-E+4 \omega_{n}\right| .
$$

Hence, at least one of the two following conditions on $\omega$ must be satisfied:

- $\left|\omega_{n-1}+\frac{E^{\prime}-E}{4}\right| \leqslant C e^{-L^{\beta} / 8}, \quad(I V)$

- $\left|\omega_{n}+\frac{E^{\prime}-E}{4}\right| \leqslant C e^{-L^{\beta} / 8}$.

From $(I)-(I V)$, Lemma 3.11 follows.

Lemmata 3.10 and 3.11 yield that if we consider any 4 consecutive interior points of $J$, we obtain at least one condition of the types $(i)-(i i i)$. Hence, the random variables $\left\{\omega_{j}\right\}_{j \in \Lambda}$ must satisfy at least $|J| / 8=c L^{\beta}$ conditions of the types $(i)-(i i i)$. From the fact that $\omega_{n}$ are i.i.d. and possess a bounded density, the conditions $(i)-($ iii $)$ imply that the event $\left(^{*}\right)$ defined in Lemma 3.3 can occur for a given partition $\mathcal{P}$ and $\mathcal{Q}$ with a probability at most $e^{-c L^{2 \beta}}$ for some $c>0$. Hence,

$$
\mathbb{P}^{*} \leqslant 2^{L} e^{-c L^{2 \beta}} \leqslant e^{-\widetilde{c} L^{2 \beta}} \text { with } 0<\widetilde{c}<c
$$

as the number of partitions is bounded by $2^{L}$ and $\beta>1 / 2$.

We therefore have Lemma 3.3 proved. 
Remark 3.12. Thanks to the equality (3.3), it is not hard to derive the following estimate for the model (1.1),

$$
\frac{\Delta E}{2 \beta_{0}}|\Lambda|^{-1 / 2} \leqslant\left\|\nabla_{\omega}\left(E(\omega)-E^{\prime}(\omega)\right)\right\| \leqslant\left\|\nabla_{\omega}\left(E(\omega)-E^{\prime}(\omega)\right)\right\|_{1}
$$

provided that $|E(\omega)-E|+\left|E^{\prime}(\omega)-E^{\prime}\right| \leqslant e^{-L^{\beta}}$ and $\Delta E=\left|E-E^{\prime}\right|$.

The above estimate reads that the $l^{1}$-distance of the gradients of $E(\omega)$ and $E^{\prime}(\omega)$ is bounded from below by a positive term that is polynomially small w.r.t. the length of the interval $\Lambda$.

Now, let $c_{1}=c_{2}$ in Lemma 3.3.

Under the hypotheses in Lemma 3.3, the estimate (3.33) implies that, for any $\left\{\omega_{j}\right\}_{j \in \Lambda}$ belonging to the event $(*)$,

$$
\frac{\Delta E}{2 \beta_{0}}|\Lambda|^{-1 / 2} \leqslant C e^{-c|\Lambda|^{\beta}}
$$

which is impossible when $|\Lambda|$ sufficiently large. Hence, for $c_{1}=c_{2}, \mathbb{P}^{*}$ is equal to 0 .

Finally, we would like to note that an estimate like (3.33) for the discrete Anderson model only holds true for two distinct energies sufficiently far apart from each other. Moreover, that kind of estimate enable us to prove the decorrelation estimate for the discrete Anderson model in any dimension (c.f. Lemma 2.4 in [8]). But it is not the case for the model (1.1).

\section{Comment on the LOWer Bound of the R.V.'S}

In this section, we want to discuss how to relax the hypothesis on the lower bound of random variables $\left\{\omega_{j}\right\}_{j \in \mathbb{Z}}$.

Assume that all r.v.'s $\omega_{j}$ are only non-negative instead of being bounded from below by a positive constant. Precisely, assume that $\omega_{j} \in\left[0, \beta_{0}\right] \forall j \in \mathbb{Z}$. In order to carry out our proof, we have to assume an extra condition on the function of distribution $F(t)$ of r.v.'s $\omega_{j}$ :

$$
F(t):=\mathbb{P}\left(\omega_{j} \leqslant t\right) \leqslant e^{-t^{-\eta}}
$$

for all small positive $t$, where $\eta$ is some positive number.

Without loss of generality, let $\eta=1$. The condition (4.1) means that the distribution $F(t)$ is exponentially small in a neighborhood of 0 .

Now, let $\Lambda=[-L, L]$ be an interval in $\mathbb{Z}$, we have

$$
\mathbb{P}\left(\exists \omega_{\gamma} \leqslant e^{-(\log L)^{\delta}} \text { with } \gamma \in \Lambda\right) \leqslant(2 L+1) e^{-e^{(\log L)^{\delta}}} .
$$

Note that the right hand side of (4.2) converges to 0 as $L \rightarrow \infty$. Here $\delta$ is a fixed number in $(0,1)$.

Hence, with a probability greater than or equal to $1-(2 L+1) e^{-e^{(\log L)^{\delta}}}$, we have

$$
\omega_{j} \geq e^{-(\log L)^{\delta}}>0 \forall j \in[-L, L] .
$$


We will use (4.3) to prove the following "lower bound" for normalized eigenvectors of $H_{\omega}(\Lambda)$.

Lemma 4.1. Pick $\beta \in(1 / 2,1)$ and a fixed number $\epsilon \in(0, \beta)$. Let $\Lambda=$ $[-L, L]$ be a large cube in $\mathbb{Z}$. Suppose that $E(\omega)$ is an eigenvalue of $H_{\omega}(\Lambda)$ and $u:=u(\omega)$ is its associated normalized eigenvector.

Then, with a probability greater than or equal to $1-(2 L+1) e^{-e^{(\log L)^{\delta}}}$, there exists a point $k_{0}$ in $\Lambda$ such that

$$
u^{2}(k)+u^{2}(k+1) \geqslant e^{-L^{\beta} / 2}
$$

for all $\left|k-k_{0}\right| \leqslant \frac{1}{4} L^{\beta-\epsilon}$ as $L$ large enough.

Proof of Lemma 4.1. Consider $\left\{\omega_{j}\right\}_{j \in \Lambda}$ such that (4.3) holds true. Using the same notations and proceed as in Lemma 3.2, for $n, m \in \Lambda$, we have

$$
v(n)=T(n, E(\omega)) \cdots T(n-m+1, E(\omega)) v(m)
$$

where $T(n, E(\omega))$ and $v(n)$ are the transfer matrix and column vector defined in the proof of Lemma 3.2. Thanks to (4.3), the transfer matrices $T(n, E(\omega))$ are well defined and invertible. Moreover, they and their inverse matrices are bounded by $C_{L}:=e^{c(\log L)^{\delta}}$ where $c>0$ depends only on $\beta_{0}$.

Thus,

$$
\|v(n)\| \leqslant C_{L}^{|n-m|}\|v(m)\|=e^{c(\log L)^{\delta}|n-m|}\|v(m)\|
$$

where $n, m \in \Lambda$.

Assume that $\left\|v\left(k_{0}\right)\right\|$ is the maximum of $\|v(n)\|$. Hence,

$$
\left\|v\left(k_{0}\right)\right\| \geq \frac{1}{\sqrt{L}}
$$

as $u$ is a normalized vector.

Pick $\kappa>0$ a fixed number and consider integers $k$ such that $\left|k-k_{0}\right| \leqslant \kappa L^{\beta-\epsilon}$. From (4.4) and (4.5), we have the following inequality

$$
\|v(k)\| \geqslant \frac{1}{\sqrt{L}} e^{-c(\log L)^{\delta}\left|k-k_{0}\right|} \geq \frac{1}{\sqrt{L}} e^{-c \kappa(\log L)^{\delta} L^{\beta-\epsilon}} \geq e^{-\kappa L^{\beta}}
$$

when $L$ is sufficiently large.

Hence, by choosing $\kappa=1 / 4$, we have

$$
u^{2}(k)+u^{2}(k+1) \geqslant e^{-L^{\beta} / 2}
$$

which completes the Lemma 4.1 .

Roughly speaking, we obtained almost the same "lower bound" for the normalized eigenvectors of finite volume operators, but with a good probability instead of the probability 1 as in Lemma 3.2.

Now, let $\beta$ be a fixed number in the interval $(1 / 2,1)$.

One the one hand, thanks to Lemma 4.1, the argument in proof of Theorem 3.3 still works out. In deed, in this case, we can proceed as in the proof 
of Lemma 3.3 to obtain at least $c L^{\beta-\epsilon}$ restrictions on r.v.'s $\omega$. Hence, the upper bound for the probability $\mathbb{P}^{*}$ in Lemma 3.3 is now

$$
B:=2^{L}\left(e^{-\widetilde{c} L^{\beta}}\right)^{c L^{\beta-\epsilon}}=2^{L} e^{-c L^{2 \beta-\epsilon}} .
$$

If we choose $\epsilon$ in Lemma 4.1 small enough such that $2 \beta-\epsilon>1$, the upper bound $B$ is exponentially small w.r.t. $L$. Hence, the Lemma 3.3 still holds true.

On the other hand, we observe that the term $(2 L+1) e^{-e^{(\log L)^{\delta}}}$ (the upper bound of the probability that (4.3) fails) is negligible compared to the $C(l / L)^{2} e^{(\log L)^{\beta}}$ (the right hand side of the decorrelation estimate in Theorem 1.2) as $L$ large. Hence, we obtain again the decorrelation estimate. In other words, Theorem 1.2 and Theorem 1.3 still hold true in this case.

\section{More than two Distinct EnERGies}

In this section, we would like to show that, following an argument in [8], we can use Theorem 1.2 to prove the asymptotic independence for any fixed number of point processes.

Theorem 5.1. For a fixed number $n \geqslant 2$, consider a finite sequence of fixed, positive energies $\left\{E_{i}\right\}_{1 \leqslant i \leqslant n}$ in the localized regime such that $\nu\left(E_{i}\right)>0$ for all $1 \leq i \leq n$.

Then, as $|\Lambda| \rightarrow+\infty, n$ point processes $\Xi\left(\xi, E_{i}, \omega, \Lambda\right)$ defined as in (1.3) converge weakly to $n$ independent Poisson processes.

Proof of Theorem 5.1. We will prove in detail the case of $n=3$ with three distinct, positive energies $E, E^{\prime}, E^{\prime \prime}$.

Consider non-empty compact intervals $\left(U_{j}\right)_{1 \leqslant j \leqslant J},\left(U_{j}^{\prime}\right)_{1 \leqslant j \leqslant J^{\prime}},\left(U_{j}^{\prime \prime}\right)_{1 \leqslant j \leqslant J^{\prime \prime}}$ in $\mathbb{R}$ and integers $\left(k_{j}\right)_{1 \leqslant j \leqslant J},\left(k_{j}^{\prime}\right)_{1 \leqslant j \leqslant J^{\prime}},\left(k_{j}^{\prime \prime}\right)_{1 \leqslant j \leq J^{\prime \prime}}$ as in Theorem 1.3.

Using notations in [8], one picks $L$ and $l$ such that $(2 L+1)=(2 l+1)\left(2 l^{\prime}+1\right)$ where $c L^{\alpha} \leqslant l \leqslant L^{\alpha} / c$ for some $\alpha \in(0,1)$ and $c>0$. Then, one decomposes

$$
\Lambda:=[-L, L]=\bigcup_{|\gamma| \leqslant l^{\prime}} \Lambda_{l}(\gamma)
$$

where $\Lambda_{l}(\gamma):=(2 l+1) \gamma+\Lambda_{l}$.

Next, for $\Lambda^{\prime} \subset \Lambda, U \subset \mathbb{R}$ and $E>0$, one defines the following Bernoulli r.v.

$$
X\left(E, U, \Lambda^{\prime}\right):= \begin{cases}1 & \text { if } H_{\omega}\left(\Lambda^{\prime}\right) \text { has at least one eigenvalue } \\ & \text { in } E+(\nu(E)|\Lambda|)^{-1} U \\ 0 & \text { otherwise }\end{cases}
$$

and put $\Sigma(E, U, l):=\sum_{|\gamma| \leqslant l^{\prime}} X\left(E, U, \Lambda_{l}(\gamma)\right)$.

First of all, [Lemma 3.2, [8]] is the first ingredient of the proof which tell us that we can actually reduce our problem to consider eigenvalues of finitevolume operators restricted on much smaller intervals. This lemma is still true in the $n$-energy case for all $n \geq 2$. 
Hence, to complete the proof of the stochastic independence w.r.t. three processes, one only need show that the quantity

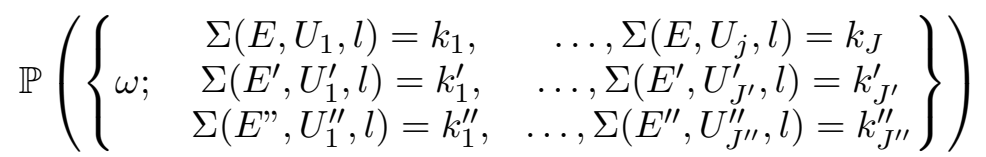

should be approximated by the product

$$
\begin{aligned}
& \mathbb{P}\left(\left\{\begin{array}{ccc}
\Sigma\left(E, U_{1}, l\right)= & k_{1} \\
\omega ; & \vdots & \vdots \\
\Sigma\left(E, U_{j}, l\right)= & k_{J}
\end{array}\right\}\right) \times \mathbb{P}\left(\left\{\begin{array}{ccc} 
& \Sigma\left(E^{\prime}, U_{1}^{\prime}, l\right)= & k_{1}^{\prime} \\
\omega ; & \vdots & \vdots \\
& \Sigma\left(E^{\prime}, U_{J^{\prime}}^{\prime}, l\right)= & k_{J^{\prime}}^{\prime}
\end{array}\right\}\right)
\end{aligned}
$$

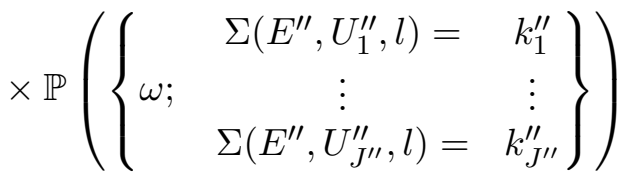

as $L$ goes to infinity. Indeed, if the above statement is proved, Theorem 1.1 and Lemma 3.2 in [8] implies Theorem 5.1.

By a standard criterion of the convergence of point processes (c.f. e.g. Theorem 11.1.VIII, [3]), the above statement holds true if the following quantity vanishes for all real numbers $t_{j}, t_{j^{\prime}}^{\prime}, t_{j^{\prime \prime}}^{\prime \prime}$ when $L$ goes to infinity:

$$
\begin{aligned}
& \mathbb{E}\left(e^{-\sum_{j=1}^{J} t_{j} \Sigma\left(E, U_{j}, l\right)-\sum_{j^{\prime}=1}^{J^{\prime}} t_{j^{\prime}}^{\prime} \Sigma\left(E^{\prime}, U_{j^{\prime}}^{\prime}, l\right)-\sum_{j^{\prime \prime}=1}^{J^{\prime \prime}} t_{j^{\prime \prime}}^{\prime \prime} \Sigma\left(E^{\prime \prime}, U_{j^{\prime \prime}}^{\prime \prime}, l\right)}\right)- \\
& \mathbb{E}\left(e^{-\sum_{j=1}^{J} t_{j} \Sigma\left(E, U_{j}, l\right)}\right) \mathbb{E}\left(e^{-\sum_{j^{\prime}=1}^{J^{\prime}} t_{j^{\prime}}^{\prime} \Sigma\left(E^{\prime}, U_{j^{\prime}}^{\prime}, l\right)}\right) \mathbb{E}\left(e^{-\sum_{j^{\prime \prime}=1}^{J^{\prime \prime}} t_{j^{\prime \prime}}^{\prime \prime} \Sigma\left(E^{\prime \prime}, U_{j^{\prime \prime}}^{\prime \prime}, l\right)}\right) .
\end{aligned}
$$

From the fact that $\{\Lambda(\gamma)\}_{|\gamma| \leqslant l^{\prime}}$ are pairwise disjoint intervals, operators $\left\{H_{\omega}(\Lambda(\gamma))\right\}_{|\gamma| \leqslant l^{\prime}}$ are independent operator-valued r.v.'s. We thus have

$$
\begin{aligned}
& \mathbb{E}\left(e^{-\sum_{j=1}^{J} t_{j} \Sigma\left(E, U_{j}, l\right)-\sum_{j^{\prime}=1}^{J^{\prime}} t_{j^{\prime}}^{\prime} \Sigma\left(E^{\prime}, U_{j^{\prime}}^{\prime}, l\right)-\sum_{j^{\prime \prime}=1}^{J^{\prime \prime}} t_{j^{\prime \prime}}^{\prime \prime} \Sigma\left(E^{\prime \prime}, U_{j^{\prime \prime}}^{\prime \prime}, l\right)}\right) \\
& =\mathbb{E}\left|\prod_{|\gamma| \leqslant l^{\prime}} e^{-\sum_{j} t_{j} X\left(E, U_{j}, \Lambda_{l}(\gamma)\right)-\sum_{j^{\prime}} t_{j^{\prime}}^{\prime} X\left(E^{\prime}, U_{j^{\prime}}^{\prime}, \Lambda_{l}(\gamma)\right)-\sum_{j^{\prime \prime}} t_{j^{\prime \prime}}^{\prime \prime} X\left(E^{\prime \prime}, U_{j^{\prime \prime}}^{\prime \prime}, \Lambda_{l}(\gamma)\right)}\right| \\
& =\prod_{|\gamma| \leqslant l^{\prime}} \mathbb{E}\left|e^{-\sum_{j} t_{j} X\left(E, U_{j}, \Lambda_{l}(\gamma)\right)-\sum_{j^{\prime}} t_{j^{\prime}}^{\prime} X\left(E^{\prime}, U_{j^{\prime}}^{\prime}, \Lambda_{l}(\gamma)\right)-\sum_{j^{\prime \prime}} t_{j^{\prime \prime}}^{\prime \prime} X\left(E^{\prime \prime}, U_{j^{\prime \prime}}^{\prime \prime}, \Lambda_{l}(\gamma)\right)}\right| .
\end{aligned}
$$

Our goal is to approximate terms of the form

$$
\mathbb{E}\left(e^{-\sum_{j}^{J} t_{j} X\left(E, U_{j}, \Lambda_{l}(\gamma)\right)-\sum_{j^{\prime}}^{J^{\prime}} t_{j^{\prime}}^{\prime} X\left(E^{\prime}, U_{j^{\prime}}^{\prime}, \Lambda_{l}(\gamma)\right)-\sum_{j^{\prime \prime}}^{J^{\prime \prime}} t_{j^{\prime \prime}}^{\prime \prime} X\left(E^{\prime \prime}, U_{j^{\prime \prime}}^{\prime \prime}, \Lambda_{l}(\gamma)\right)}\right)
$$

by the product

$$
\prod_{j}^{J} \mathbb{E} e^{-t_{j} X\left(E, U_{j}, \Lambda_{l}(\gamma)\right)} \prod_{j^{\prime}}^{J^{\prime}} \mathbb{E} e^{-t_{j} X\left(E^{\prime}, U_{j^{\prime}}^{\prime}, \Lambda_{l}(\gamma)\right)} \prod_{j^{\prime \prime}}^{J^{\prime \prime}} \mathbb{E} e^{-t_{j^{\prime \prime}}^{\prime \prime} X\left(E^{\prime \prime}, U_{j^{\prime \prime}}^{\prime \prime}, \Lambda_{l}(\gamma)\right)}
$$


as $L$ large enough with the remark that these r.v.'s are not independent. To illustrate our computation for that, we will consider just three arbitrary Bernoulli r.v.'s $X_{i}=X\left(E_{i}, U_{i}, \Lambda^{\prime}\right), i \in\{1,2,3\}$ and compute explicitly $\mathbb{E}\left(e^{\sum_{i=1}^{3} a_{i} X_{i}}\right)$.

Lemma 5.2. Let $\left\{E_{i}\right\}_{i=\overline{1,3}}$ be three positive, distinct energies in the localized regime and $\left\{U_{i}\right\}_{i=\overline{1,3}}$ be three non-empty compact intervals in $\mathbb{R}$. Assume that $\Lambda^{\prime}$ is a sub-interval of $\Lambda=[-L, L]$ such that $\left|\Lambda^{\prime}\right|=2 l+1=O\left(L^{\alpha}\right)$ for some $\alpha \in(0,1)$. Consider three Bernoulli r.v.'s $X_{i}=X\left(E_{i}, U_{i}, \Lambda^{\prime}\right)$ defined as in (5.1), we have

$$
\mathbb{E}\left(e^{\sum_{i=1}^{3} a_{i} X_{i}}\right)=\prod_{i=1}^{3} \mathbb{E}\left(e^{a_{i} X_{i}}\right)+O\left((l / L)^{1+\theta}\right) .
$$

with any $\theta \in(0,1)$ as $L$ large enough .

Proof of Lemma 5.2. Put $A:=\mathbb{E}\left(e^{\sum_{i=1}^{3} a_{i} X_{i}}\right)$, we have

$$
\begin{aligned}
A & =\mathbb{P}\left(X_{1}=X_{2}=X_{3}=0\right)+\sum_{i=1}^{3} e^{a_{i}} \mathbb{P}\left(\begin{array}{c}
X_{i}=1, \\
X_{j}=0 \forall j \neq i
\end{array}\right) \\
& +\sum_{i<j} e^{a_{i}+a_{j}} \mathbb{P}\left(\begin{array}{c}
X_{i}=X_{j}=1, \\
X_{k}=0 ; k \neq i, j
\end{array}\right)+e^{\sum_{i=1}^{3} a_{i}} \mathbb{P}\left(\bigcap_{i=1}^{3}\left\{X_{i}=1\right\}\right) .
\end{aligned}
$$

First of all, we rewrite

$$
\begin{aligned}
& \mathbb{P}\left(X_{1}=X_{2}=X_{3}=0\right)=1-\sum_{i=1}^{3} \mathbb{P}\left(\begin{array}{c}
X_{i}=1, \\
X_{j}=0 \forall j \neq i
\end{array}\right) \\
& -\sum_{i<j} \mathbb{P}\left(\begin{array}{c}
X_{i}=X_{j}=1, \\
X_{k}=0 ; k \neq i, j
\end{array}\right)-\mathbb{P}\left(\bigcap_{i=1}^{3}\left\{X_{i}=1\right\}\right)
\end{aligned}
$$

and obtain that

$$
\begin{aligned}
& A=1+\sum_{i=1}^{3}\left(e^{a_{i}}-1\right) \mathbb{P}\left(\begin{array}{c}
X_{i}=1 \\
X_{j}=0 \forall j \neq i
\end{array}\right) \\
& +\sum_{i<j}\left(e^{a_{i}+a_{j}}-1\right) \mathbb{P}\left(\begin{array}{c}
X_{i}=X_{j}=1, \\
X_{k}=0 ; k \neq i, j
\end{array}\right)+\left(e^{\sum_{i=1}^{3} a_{i}}-1\right) \mathbb{P}\left(\bigcap_{i=1}^{3}\left\{X_{i}=1\right\}\right) .
\end{aligned}
$$

Next, use

$$
\begin{aligned}
& \mathbb{P}\left(\begin{array}{c}
X_{i}=1, \\
X_{j}=0 \forall j \neq i
\end{array}\right)=\mathbb{P}\left(X_{i}=1\right)-\mathbb{P}\left(\begin{array}{c}
X_{i}=X_{i+1}=1 \\
X_{i+2}=0
\end{array}\right) \\
& -\mathbb{P}\left(\begin{array}{c}
X_{i}=X_{i-1}=1, \\
X_{i+1}=0
\end{array}\right)-\mathbb{P}\left(\bigcap_{i=1}^{3}\left\{X_{i}=1\right\}\right)
\end{aligned}
$$


to get

$$
\begin{aligned}
& A=1+\sum_{i=1}^{3}\left(e^{a_{i}}-1\right) \mathbb{P}\left(X_{i}=1\right)+\sum_{i<j}\left(e^{a_{i}}-1\right)\left(e^{a_{j}}-1\right) \mathbb{P}\left(\begin{array}{c}
X_{i}=X_{j}=1, \\
X_{k}=0 k \neq i, j
\end{array}\right) \\
& +\left(e^{\sum_{i=1}^{3} a_{i}}-1-\sum_{i=1}^{3}\left(e^{a_{i}}-1\right)\right) \mathbb{P}\left(\bigcap_{i=1}^{3}\left\{X_{i}=1\right\}\right) .
\end{aligned}
$$

Using a similar expansion for all terms of the form $\mathbb{P}\left(\begin{array}{c}X_{i}=X_{j}=1, \\ X_{k}=0 k \neq i, j\end{array}\right)$, we obtain the following formula

$$
A=1+\sum_{i=1}^{3}\left(e^{a_{i}}-1\right) \mathbb{P}\left(X_{i}=1\right)+\sum_{i<j}\left(e^{a_{i}}-1\right)\left(e^{a_{j}}-1\right) \mathbb{P}\left(X_{i}=X_{j}=1\right)
$$

$$
+\prod_{i=1}^{3}\left(e^{a_{i}}-1\right) \mathbb{P}\left(\bigcap_{i=1}^{3}\left\{X_{i}=1\right\}\right)
$$

On the other hand, from the observation that

$$
\mathbb{E} e^{a_{j} X_{j}}=1+\left(e^{a_{j}}-1\right) \mathbb{P}\left(X_{j}=1\right) \forall j=\{1,2,3\},
$$

we multiply the three equalities above to get

$$
\prod_{i=1}^{3} \mathbb{E}\left(e^{a_{i} X_{i}}\right)=1+\sum_{i=1}^{3}\left(e^{a_{i}}-1\right) \mathbb{P}\left(X_{i}=1\right)
$$

$$
+\sum_{i<j}\left(e^{a_{i}}-1\right)\left(e^{a_{j}}-1\right) \mathbb{P}\left(X_{i}=1\right) \mathbb{P}\left(X_{j}=1\right)+\prod_{i=1}^{3}\left(e^{a_{i}}-1\right) \mathbb{P}\left(X_{i}=1\right) .
$$

Hence, thanks to (5.4) and (5.5), we have

$$
\begin{aligned}
& \mathbb{E}\left(e^{\sum_{i=1}^{3} a_{i} X_{i}}\right)-\prod_{i=1}^{3} \mathbb{E}\left(e^{a_{i} X_{i}}\right)= \\
& \sum_{i<j}\left(e^{a_{i}}-1\right)\left(e^{a_{j}}-1\right)\left[\mathbb{P}\left(X_{i}=X_{j}=1\right)-\mathbb{P}\left(X_{i}=1\right) \mathbb{P}\left(X_{j}=1\right)\right] \\
& \left.+\prod_{i=1}^{3}\left(e^{a_{i}}-1\right)\left[\bigcap_{j=1}^{3}\left\{X_{j}=1\right\}\right)-\prod_{j=1}^{3} \mathbb{P}\left(X_{j}=1\right)\right] .
\end{aligned}
$$

Theorem 1.2 and Theorem 2.1 yield, for any $\theta \in(0,1)$,

$$
\begin{gathered}
\mathbb{P}\left(X_{i}=X_{j}=1\right)+\mathbb{P}\left(X_{i}=1\right) \mathbb{P}\left(X_{j}=1\right) \\
\leqslant C(l / L)^{2}\left(e^{(\log L)^{\beta}}+1\right) \leqslant C(l / L)^{1+\theta}
\end{gathered}
$$


and

$$
\begin{aligned}
& \mathbb{P}\left(\bigcap_{i=1}^{3}\left\{X_{i}=1\right\}\right)+\mathbb{P}\left(X_{1}=1\right) \mathbb{P}\left(X_{2}=1\right) \mathbb{P}\left(X_{3}=1\right) \\
& \leqslant C(l / L)^{2}\left(e^{(\log L)^{\beta}}+(l / L)\right) \leqslant C(l / L)^{1+\theta}
\end{aligned}
$$

with $L$ large enough. Note that $C>0$ is a constant depending only on positive, fixed energies $\left\{E_{i}\right\}_{i=1}^{3}$.

From (5.6)-(5.8), Lemma 5.2 follows.

It is easy to see that the computation in Lemma 5.2 can apply to any finite number of Bernoulli r.v.'s defined as in (5.1). More precisely, for each $|\gamma| \leqslant l^{\prime}$, we have

$$
\begin{aligned}
& \mathbb{E}\left(e^{-\sum_{j} t_{j} X\left(E, U_{j}, \Lambda_{l}(\gamma)\right)-\sum_{j^{\prime}} t_{j^{\prime}}^{\prime} X\left(E^{\prime}, U_{j^{\prime}}^{\prime}, \Lambda_{l}(\gamma)\right)-\sum_{j^{\prime \prime}} t_{j^{\prime \prime}}^{\prime \prime} X\left(E^{\prime \prime}, U_{j^{\prime \prime}}^{\prime \prime}, \Lambda_{l}(\gamma)\right)}\right) \\
& =\prod_{j}^{J} \mathbb{E} e^{-t_{j} X\left(E, U_{j}, \Lambda_{l}(\gamma)\right)} \prod_{j^{\prime}}^{J^{\prime}} \mathbb{E} e^{-t_{j^{\prime}}^{\prime} X\left(E^{\prime}, U_{j^{\prime}}^{\prime}, \Lambda_{l}(\gamma)\right)} \prod_{j^{\prime \prime}}^{J^{\prime \prime}} \mathbb{E} e^{-t_{j^{\prime \prime}}^{\prime \prime} X\left(E^{\prime \prime}, U_{j^{\prime \prime}}^{\prime \prime}, \Lambda_{l}(\gamma)\right)} \\
& \times\left(1+O(l / L)^{1+\theta}\right) .
\end{aligned}
$$

On the other hand, we also have similar formulas for $\mathbb{E} e^{-\sum_{j} t_{j} X\left(E, U_{j}, \Lambda_{l}(\gamma)\right)}$, $\mathbb{E} e^{-\sum_{j^{\prime}} t_{j^{\prime}}^{\prime} X\left(E^{\prime}, U_{j}^{\prime}, \Lambda_{l}(\gamma)\right)}$ and $\mathbb{E} e^{-\sum_{j^{\prime \prime}} t_{j}^{\prime \prime} X\left(E^{\prime \prime}, U_{j^{\prime \prime}}^{\prime \prime}, \Lambda_{l}(\gamma)\right)}$.

We therefore infer that:

$$
\begin{aligned}
& \mathbb{E}\left(e^{-\sum_{j} t_{j} X\left(E, U_{j}, \Lambda_{l}(\gamma)\right)-\sum_{j^{\prime}} t_{j^{\prime}}^{\prime} X\left(E^{\prime}, U_{j^{\prime}}^{\prime}, \Lambda_{l}(\gamma)\right)-\sum_{j^{\prime \prime}} t_{j^{\prime \prime}}^{\prime \prime} X\left(E^{\prime \prime}, U_{j^{\prime \prime}}^{\prime \prime}, \Lambda_{l}(\gamma)\right)}\right) \\
& =\mathbb{E} e^{-\sum_{j} t_{j} X\left(E, U_{j}, \Lambda_{l}(\gamma)\right)} \mathbb{E} e^{-\sum_{j^{\prime}} t_{j^{\prime}}^{\prime} X\left(E^{\prime}, U_{j^{\prime}}^{\prime}, \Lambda_{l}(\gamma)\right)} \mathbb{E} e^{-\sum_{j^{\prime \prime}} t_{j^{\prime \prime}}^{\prime \prime} X\left(E^{\prime \prime}, U_{j^{\prime \prime}}^{\prime \prime}, \Lambda_{l}(\gamma)\right)} \\
& \times\left(1+O(l / L)^{1+\theta}\right) .
\end{aligned}
$$

We have an observation that $|\gamma| \leqslant l^{\prime}$ where $l^{\prime}=O(L / l)$. Hence, by multiplying all above equalities side by side over $|\gamma| \leq l^{\prime}$, we obtain that:

$$
\mathbb{E}\left(e^{-\sum_{j=1}^{J} t_{j} \Sigma\left(E, U_{j}, l\right)-\sum_{j^{\prime}=1}^{J^{\prime}} t_{j^{\prime}}^{\prime} \Sigma\left(E^{\prime}, U_{j^{\prime}}, l\right)-\sum_{j^{\prime \prime}=1}^{J^{\prime \prime}} t_{j^{\prime \prime}}^{\prime \prime} \Sigma\left(E^{\prime \prime}, U_{j^{\prime \prime}}, l\right)}\right)
$$

is equal to the product of

$$
\mathbb{E}\left(e^{-\sum_{j=1}^{J} t_{j} \Sigma\left(E, U_{j}, l\right)}\right) \mathbb{E}\left(e^{-\sum_{j^{\prime}=1}^{J^{\prime}} t_{j^{\prime}}^{\prime} \Sigma\left(E^{\prime}, U_{j^{\prime}}, l\right)}\right) \mathbb{E}\left(e^{-\sum_{j^{\prime \prime}=1}^{J^{\prime \prime}} t_{j^{\prime \prime}}^{\prime \prime} \Sigma\left(E^{\prime \prime}, U_{j^{\prime \prime}}, l\right)}\right)
$$

and an error term of the form $\left(1+x^{1+\theta}\right)^{1 / x}$ with $x=O(l / L)$.

Note that the above error term tends to 1 as $L$ goes to infinity. Hence, the stochastic independence for three point processes w.r.t. three distinct energies is proved. Finally, it is not hard to see that we can adapt this proof for $n$-energy case with any $n \geq 2$. 


\section{APPENDix A.}

Compute the determinant of matrix $A_{0}$ in (3.31). Put $A_{0}=\left(a_{i j}\right)$, we'll give here some details of computing the determinant of $A_{0}$ by hand (A mathematical software like Maple or Mathematica might be useful for checking the final result of this computation).

First, expand this determinant by its sixth and last column and then by its first column to get

$$
\left|\operatorname{det} A_{0}\right|=\omega_{n-2}\left|\operatorname{det} B_{0}\right|
$$

where $B_{0}$ is the $7 \times 7$ matrix defined by

$$
\left(\begin{array}{ccccccc}
1 & -1 & 0 & 0 & 1 & -1 & 0 \\
0 & 1 & -1 & 0 & 0 & 1 & -1 \\
-\omega_{n-1} & \omega_{n-1}+\omega_{n}-E & -\omega_{n} & 0 & 0 & 0 & 0 \\
0 & \frac{E}{E^{\prime}} & 0 & 0 & 0 & -1 & 0 \\
0 & -\omega_{n} & \omega_{n}+\omega_{n+1}-E & -\omega_{n+1} & 0 & 0 & 0 \\
0 & \omega_{n} & \omega_{n+1}-\omega_{n} & -\omega_{n+1} & 0 & 0 & E^{\prime} \\
\frac{E}{E^{\prime}} & 0 & 0 & 0 & -1 & 0 & 0
\end{array}\right)
$$

Now, we compute the determinant of $B_{0}$.

Take the sixth row minus the fifth row and take the first row plus the last row. Next, multiply the second row by $E^{\prime}$ and take it plus the sixth row. Finally, expand the determinant of $B_{0}$ by the forth, the fifth and the last column to get

$$
\left|\operatorname{det} A_{0}\right|=\omega_{n-2} \omega_{n+1}\left|\operatorname{det} C_{0}\right|
$$

where $C_{0}$ is the $4 \times 4$ matrix defined by

$$
\left(\begin{array}{cccc}
1+\frac{E}{E^{\prime}} & -1 & 0 & -1 \\
0 & E^{\prime}+2 \omega_{n} & E-E^{\prime}-2 \omega_{n} & E^{\prime} \\
-\omega_{n-1} & \omega_{n-1}+\omega_{n}-E & -\omega_{n} & 0 \\
0 & \frac{E}{E^{\prime}} & 0 & -1
\end{array}\right)
$$

Finally, by an explicit computation for the determinant of $C_{0}$, we obtain that

$$
\left|\operatorname{det} A_{0}\right|=\frac{4 E}{E^{\prime}}\left(E+E^{\prime}\right) \omega_{n-2} \omega_{n+1}\left|\omega_{n}+\frac{E^{\prime}-E}{4}\right| .
$$

Compute the determinant of matrix $A_{1}$ in (3.32). The determinant of $A_{1}$ can be computed as follows: First, expand this determinant by its sixth and last column and then by its fifth and first column to get

$$
\left|\operatorname{det} A_{1}\right|=\omega_{n-2} \omega_{n+1}\left|\operatorname{det} B_{1}\right|
$$


where $B_{1}$ is the $6 \times 6$ matrix defined by

$$
\left(\begin{array}{cccccc}
1 & -1 & 0 & -1 & 1 & 0 \\
0 & 1 & -1 & 0 & 1 & -1 \\
-\omega_{n-1} & \omega_{n-1}+\omega_{n}-E & -\omega_{n} & 0 & 0 & 0 \\
\omega_{n-1} & \omega_{n}-\omega_{n-1} & -\omega_{n} & 0 & -E^{\prime} & 0 \\
0 & 0 & \frac{E}{E^{\prime}} & 0 & 0 & -1 \\
-\frac{E}{E^{\prime}} & 0 & 0 & -1 & 0 & 0
\end{array}\right)
$$

Second, take the first row of matrix B minus its last row and take the second row minus the fifth row, then expand the determinant of $B_{1}$ by its forth and sixth column to obtain

$$
\left|\operatorname{det} A_{1}\right|=\omega_{n-2} \omega_{n+1}\left|\operatorname{det} C_{1}\right|
$$

where $C_{1}$ is the following $4 \times 4$ matrix

$$
\left(\begin{array}{cccc}
1+\frac{E}{E^{\prime}} & -1 & 0 & 1 \\
0 & 1 & -1-\frac{E}{E^{\prime}} & 1 \\
-\omega_{n-1} & \omega_{n-1}+\omega_{n}-E & -\omega_{n} & 0 \\
\omega_{n-1} & \omega_{n}-\omega_{n-1} & -\omega_{n} & -E^{\prime}
\end{array}\right)
$$

Finally, by an explicit computation for the determinant of the matrix $C_{1}$, we infer that

$$
\left|\operatorname{det} A_{1}\right|=\frac{4 E}{E^{\prime}} \omega_{n-2} \omega_{n+1}\left|\omega_{n-1} \omega_{n}-\frac{\left(E+E^{\prime}\right)^{2}}{4}\right| .
$$

Acknowledgements. This work would have been impossible without great help of my advisor, Prof. Frédéric Klopp. The author gratefully acknowledges the collaboration and various discussions with him. I am grateful for some corrections and helpful suggestions made by an anonymous referee which definitely improved the presentation of this work.

\section{REFERENCES}

[1] Alexander Figotin and Abel Klein, Localization of electromagnetic and acoustic waves in random media. Lattice models, J. Statist. Phys., 76(3- 4):9851003, 1994.

[2] Michael Aizenman, Jeffrey H.Schenker, Roland M. Friedrich, and Dirk Hundertmark, Finite-volume fractional-moment criteria for Anderson localization, Comm. Math. Phys., 224(1):219-253, 2001. Dedicated to Joel L. Lebowitz.

[3] D.J.Daley, D.Vere-Jones, An Introduction to the Theory of Point Processes. Vol. II. General Theory and Structure, 2nd edition, Probability and its applications (New York). Springer, New York (2008)

[4] Peter D. Hislop, Lectures on random Schrödinger operators, In Fourth Summer School in Analysis and Mathematical Physics, volume 476 of Contemp. Math., pages 41131. Amer. Math. Soc., Providence, RI, 2008.

[5] Dong Miao, Eigenvalue statistics for lattice Hamiltonian of off-diagonal disorder, J. Stat. Phys (2011), 143: 509-522 DOI 10.1007/s10955-011-0190-2. 
[6] Nariyuki Minami, Local fluctuation of the spectrum of a multidimensional Anderson tight binding model, Comm. Math. Phys. Vol. 177, 709-725 (1996).

[7] François Germinet and Frédéric Klopp, Spectral statistics for random Schrödinger operators in the localized regime, To appear in Jour. Eur. Math. Soc.

[8] Frédéric Klopp, Decorrelation estimates for the eigenvalues of the discrete Anderson model in the localized regime, Comm. Math. Phys. Vol. 303, pp. 233-260 (2011).

[9] Tosio Kato, Perturbation Theory for Linear Operators. Springer-Verlag, Berlin, 1995. Reprint of 1980 edition.

(Trinh Tuan Phong) Laboratoire Analyse, Géométrie et Applications,

UMR 7539, Institut Galilée, Université Paris 13,

99 avenue J.B. Clément, 93430 Villetaneuse, France

Email: trinh@math.univ-paris13.fr 